\title{
Position Analysis of a Hybrid Serial-Parallel Manipulator in Immersion Lithography
}

\author{
Jie-jie Shao, Tong Ma, Wen-yu Chen, and Xin Fu \\ The State Key Laboratory of Fluid Power Transmission and Control, Zhejiang University, Hangzhou, Zhejiang 310027, China \\ Correspondence should be addressed to Wen-yu Chen; serverlandon@zju.edu.cn
}

Received 6 November 2014; Revised 2 March 2015; Accepted 10 March 2015

Academic Editor: Valter J. S. Leite

Copyright ( 2015 Jie-jie Shao et al. This is an open access article distributed under the Creative Commons Attribution License, which permits unrestricted use, distribution, and reproduction in any medium, provided the original work is properly cited.

\begin{abstract}
This paper proposes a novel hybrid serial-parallel mechanism with 6 degrees of freedom. The new mechanism combines two different parallel modules in a serial form. $3-\mathrm{P}\left(\mathrm{P}_{\mathrm{H}}\right)$ parallel module is architecture of 3 degrees of freedom based on higher joints and specializes in describing two planes' relative pose. 3-PSP parallel module is typical architecture which has been widely investigated in recent researches. In this paper, the direct-inverse position problems of the 3-PSP parallel module in the couple mixed-type mode are analyzed in detail, and the solutions are obtained in an analytical form. Furthermore, the solutions for the direct and inverse position problems of the novel hybrid serial-parallel mechanism are also derived and obtained in the analytical form. The proposed hybrid serial-parallel mechanism is applied to regulate the immersion hood's pose in an immersion lithography system. Through measuring and regulating the pose of the immersion hood with respect to the wafer surface simultaneously, the immersion hood can track the wafer surface's pose in real-time and the gap status is stabilized. This is another exploration to hybrid serial-parallel mechanism's application.
\end{abstract}

\section{Introduction}

Immersion lithography is a more advanced semiconductor technology compared with the traditional dry lithography. Immersion technology can improve the lithography resolution to $45 \mathrm{~nm}$ or even higher by replacing the air between the wafer and bottom lens with liquid of higher refractive index [1-3]. The efficient implementation of immersion technology must be based on the successful management of immersion fluid $[4,5]$. While the wafer scans, a device called immersion hood (IH) is used to constrain and update the immersion fluid within the limited area below the lens [6, 7], as shown in Figure 1. In order to form a piece of immersion flow field without leakage and pressure fluctuation, a steady gap with specified parallelism and central distance should be formed between the IH and the wafer. Immersion hood pose regulation manipulator (IHPRM) is employed to precisely adjust and sustain the pose of the $\mathrm{IH}$ with respect to the wafer surface.

Commonly, the main structure of the IHPRM is a 3degree of freedom (DOF) parallel mechanism (PM), which adjusts the pose of the IH's lower surface with respect to the upper surface of the wafer. The other three DOFs of the $\mathrm{IH}$ are actuated by three independent screws manually during the assembling process and then fixed, as shown in Figure 1. As the pose parameters of the tiny gap (about $0.1 \mathrm{~mm}$ ) is extremely hard to obtain directly, the indirect pose measurement (with respect to the main frame) is usually applied in the immersion hood pose regulation, which performs insufficient precision in the pose regulating. On the other hand, the wafer stage adjusts the pose of the wafer with respect to the optical focus and scans simultaneously during the exposure process; thus, the pose of the immersion hood should be adjusted accordingly in the meantime. However, the kinematic problem of the parallel mechanism is often solved via numerical iterative methods which can provide only some of the possible solutions and usually need more time compared to the analytical method. Therefore, the general 3-DOF parallel mechanism is difficult to be competent in high precision pose regulation and real-time tracking.

The hybrid serial-parallel mechanism (HS-PM) which combines high precision, high rigidity, and low inertia of fully parallel mechanism and extended workspace of serial mechanism have attracted increasing attention during the past 


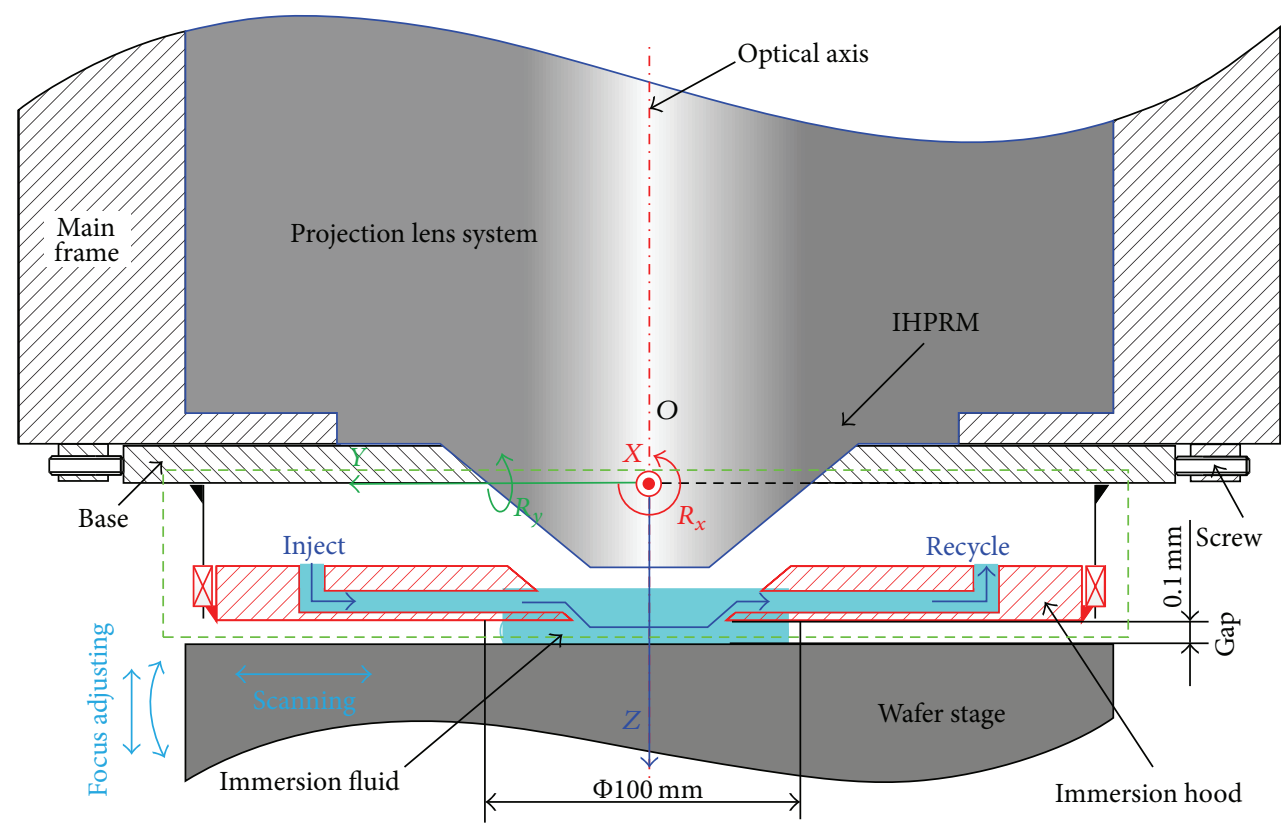

FIGURE 1: The IHPRM in immersion lithography.

several years. Waldron et al. [8] have analyzed a hybrid type of manipulator with 10 DOFs, which comprises a serial arm with 6 DOFs and a parallel micromanipulator with 4 DOFs in 1989. Naccarato et al. [9] raised the so-called variablegeometry truss manipulators which realize the combination of the parallel manipulators in a serial form in 1991. Shahinpoor [10] has studied a hybrid serial-parallel manipulator formed by two serially connected parallel manipulators and analyzed the inverse kinematic problem in 1992. Romdhane [11] proposed a Stewart platform-like hybrid serial-parallel mechanism, obtained a closed-form solution of the forward analysis problem, and analyzed the orientation workspace in 1999. Tanev [12] presented a hybrid robot with 6 DOFs and acquired the closed-form solutions for the direct and inverse position problems in 2000. Kanaan et al. [13] applied 5-DOF serial-parallel architecture in the VERNE machine tool and derived its inverse-forward kinematic equations in 2009. Ibrahim and Khalil [14] discussed the dynamic models of the hybrid robots in 2010. Zeng et al. [15, 16] analyzed the hybrid serial-parallel mechanisms with spatial multiloop kinematic chains in 2012 and put forward a design method of hybrid manipulators with kinematotropic property and deployability in 2014.

Kinematic analysis is the basic issue for the parallel mechanism studies, and the position analysis is the first step of the kinematic analysis. As the HS-PM is generally composed of several parallel mechanisms (regarded as its parallel module) with lower mobility, whether analytical form solutions can be obtained for the HS-PM's direct-inverse position problem mainly depends on whether its parallel modules can. 3-PSP is a typical parallel mechanism (PM) with 3 DOFs and has been widely discussed in recent years. In 2001, Gregorio and Parenti-Castelli [17] made the position analysis of a general 3-PSP parallel robot in an analytical form. Deng and
Li [18] obtained the 3-PSP PM's direct position solution in a numerical form by solving 9 independent equations in 2009. Hao et al. [19] analyzed the 3-PSP PM in 2009. But because the pose status was described by Tilt-Torsion angle, only the inverse position problem was solved in an analytical form. Zhang and Li [20] discussed the direct position problem of the 3-PSP PM with the screw theory in 2013, which still needs to solve 9 independent equations. Rezaei et al. [21] investigated the 3-PSP PM systematically in 2013. The directinverse position problem was divided into two modes according to the different pose parameters: the coupled mixed-type mode with $\theta, \varphi$, and $z_{p}$ and the nonpure translational mode with $x_{p}, y_{p}$, and $z_{p}$. A geometrical approach was applied to the analysis of inverse position problem in the nonpure translational mode, and a solution in an analytical form was obtained. However, the coupled mixed-type mode which is more frequently used when describing a moving platform's position and orientation was calculated in the numerical way. Currently, the analytical solutions for the direct-inverse position problems of 3-PSP PM in the coupled mixed-type mode are still absent.

In this paper, according to the high precision regulation and real-time tracking demand of the IHPRM in the immersion lithography system, a new type of hybrid serial-parallel mechanism with 6 DOFs is proposed. The new mechanism is constituted by combining two different parallel modules (including a 3-PSP module and a 3- $\underline{\mathrm{P}}\left(\mathrm{P}_{\mathrm{H}}\right)$ module) in a serial form. The 3-PSP module is employed to regulate IH's pose, while the 3- $\underline{\mathrm{P}}\left(\mathrm{P}_{\mathrm{H}}\right)$ module is adopted to detect the pose status of the IH's lower surface with respect to the wafer's upper surface.

The direct-inverse position problems of 3-PSP PM in the coupled mixed-type mode as the basis of the kinematic analysis are fully discussed in this paper. By choosing proper 


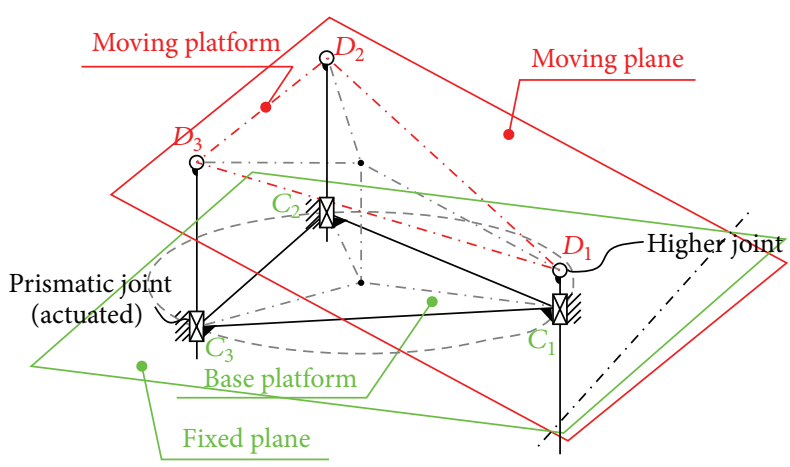

(a) The kinematic model

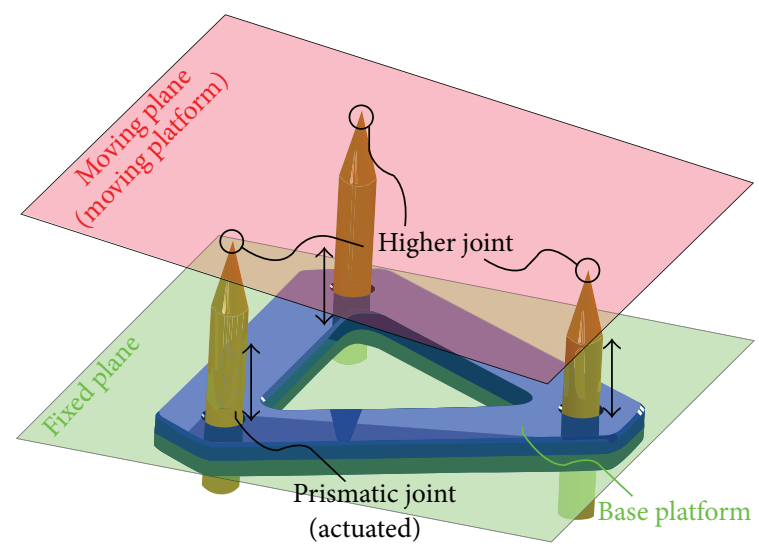

(b) The CAD model

FIgURE 2: The schematic of the 3- $\underline{\mathrm{P}}\left(\mathrm{P}_{\mathrm{H}}\right)$ parallel mechanism.

pose parameters and geometric constraints, the analytical solutions to both direct and inverse position problems are obtained. On this basis, the direct-inverse position analysis for the new hybrid serial-parallel mechanism is also discussed in detail, which acquires an analytical solution as well. Finally, this new mechanism realizes tracking IH's lower surface with respect to the wafer surface in real time. The variation of gap parameters caused by pose regulation during wafer scanning can be reduced effectively.

\section{Structure Description of the Hybrid Serial-Parallel Manipulator}

2.1. The Hybrid Serial-Parallel Mechanism. As a subsystem of immersion lithography system, IHPRM holds the IH in the proper pose and assures a stable gap formed between IH's lower surface and the wafer surface which is also suitable for fluid management. In the present application, the thickness of the gap should be adjusted within the range of $100 \pm 5 \mu \mathrm{m}$. The angle between IH's lower surface and the wafer surface cannot exceed $0.00005 \mathrm{rad}$, and the tilt direction should be in accordance with wafer scanning direction. Therefore, IHPRM must adjust poses in 3 DOFs. As the pose of the wafer surface varies during wafer scanning, the pose of the IH's lower surface should track wafer's surface in real time to sustain a steady gap. It means a necessity of the detection of the gap's status in real time. Therefore, IHPRM must satisfy 3 DOFs' detecting requirements. The pose regulation process can be simplified as the movements among three planes: the base plane is fixed, the pose of a target plane with respect to base plane varies to keep the surface of the wafer within the focus depth of the lens, and a moving plane is adjusted to keep the parallelism and the distance with plane $\varepsilon$. The delta mechanism, Stewart's platform, and other normal parallel mechanisms only adjust the pose between two platforms, which are not suitable for this application. In this paper, a novel HS-PM is designed to realize both immersion hood pose's regulation and the gap's status detection. As the new mechanism is composed of a 3- $\underline{\mathrm{P}}\left(\mathrm{P}_{\mathrm{H}}\right) \mathrm{PM}$ and a 3-PSP PM, we call it 3-PSP-P $\left(\mathrm{P}_{\mathrm{H}}\right)$ HS-PM. Combining two Stewart's platforms in a series form to adjust these three planes is obviously much more complex and redundant than the 3$\underline{\mathrm{PSP}}-\underline{\mathrm{P}}\left(\mathrm{P}_{\mathrm{H}}\right) \mathrm{HS}-\mathrm{PM}$.

2.1.1. The 3-P( $\left.P_{H}\right)$ Parallel Modular. The 3- $-\left(\mathrm{P}_{\mathrm{H}}\right) \mathrm{PM}$ consists of a base platform, a moving platform, and three chains with identical kinematic architecture, as shown in Figure 2. The moving platform, actually a moving plane, is a virtual object without definite shape. Each chain connects the moving platform to the base platform through a prismatic joint $\mathrm{P}$ and a higher joint $\left(\mathrm{P}_{\mathrm{H}}\right)$ in sequence, where the prismatic joint $\mathrm{P}$ is the active joint. The translational axis $\left(C_{i} D_{i}\right)$ of the prismatic joint $\mathrm{P}$ within the $i$ th $(i=1,2,3)$ kinematic chain is perpendicular to the base. The higher joints $\left(\mathrm{P}_{\mathrm{H} i}\right)$ are attached to the sliders moving along these axes $\left(C_{i} D_{i}\right)$, respectively, with their apexes $D_{i}$ constrained within the moving plane.

Here, the Grübler-Kutzbach criterion [22, 23] is applied to analyze the DOF of the $3-\underline{P}\left(\mathrm{P}_{\mathrm{H}}\right) \mathrm{PM}$. Consider

$$
\mathrm{M}=\lambda(n-j-1)+\sum_{i=1}^{j} f_{i}=6(5-6-1)+18=6,
$$

where $\lambda$ indicates the dimension of task space, $n$ is the number of links, $j$ is the number of joints, and $f_{i}$ stands for the DOF of joint $i$.

As shown in (1), 3- $\underline{\mathrm{P}}\left(\mathrm{P}_{\mathrm{H}}\right) \mathrm{PM}$ has 6 DOFs, which can be divided into two types. The 1 translational DOF and 2 rotational DOFs that the moving plane moves with respect to the base platform are regarded as the first type, while the 2 translational DOFs and 1 rotational DOF that the virtual moving platform moves in the moving plane are regarded as the second type. Since the moving platform is virtual and has no definite shape, its motion in the moving plane is meaningless, and the $3-\underline{P}\left(\mathrm{P}_{\mathrm{H}}\right) \mathrm{PM}$ has 3 DOFs.

2.1.2. The 3-PSP Parallel Modular. The 3-PSP PM consists of a base platform, a moving platform, and three chains with identical kinematic architecture and is a parallel mechanism 


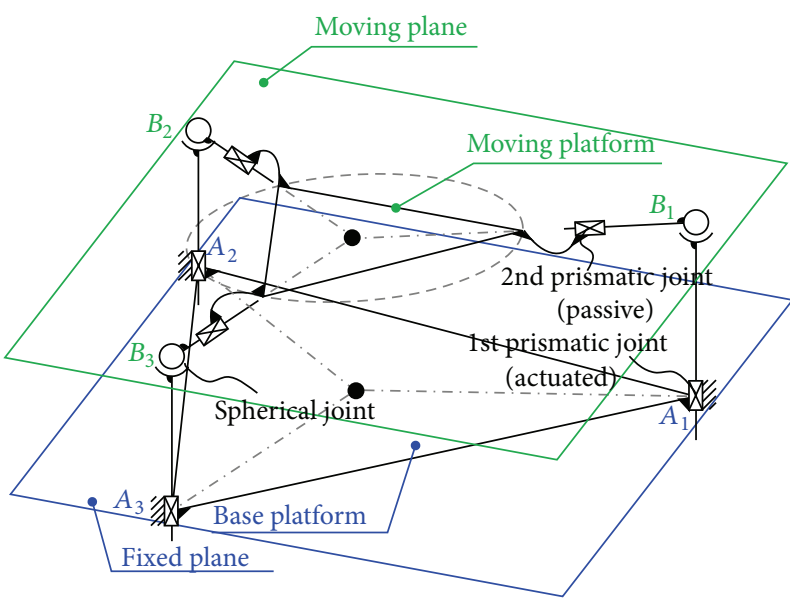

(a) The kinematic model

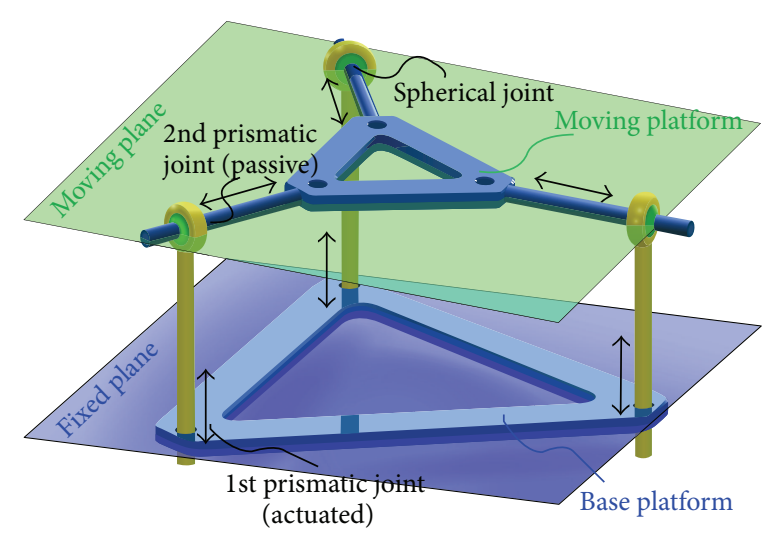

(b) The CAD model

FIGURE 3: The schematic of the 3-PSP parallel mechanism.

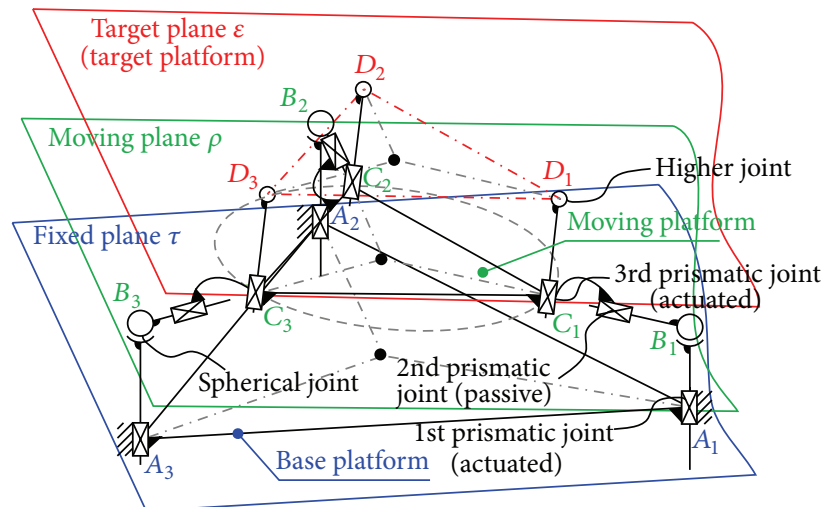

(a) The kinematic model

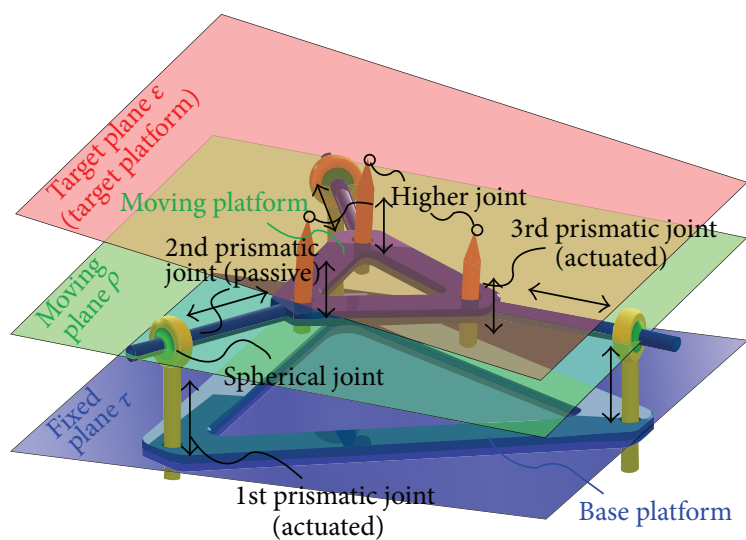

(b) The CAD model

FIgURE 4: The schematic of the HS-PM.

with 3 DOFs [24]. With reference to Figure 3, the moving platform and the base platform are connected to each other by means of three independent kinematic chains which are constituted by an active prismatic joint $\underline{P}$, a spherical joint $S$, and a passive joint $\mathrm{P}$ in sequence.

2.1.3. The Hybrid Mechanism. The novel 3-PSP- $-\mathrm{P}\left(\mathrm{P}_{\mathrm{H}}\right)$ HS$\mathrm{PM}$ combines these two parallel modules in serial form, by attaching the base platform of the $3-\underline{\mathrm{P}}\left(\mathrm{P}_{\mathrm{H}}\right) \mathrm{PM}$ to the moving platform of the 3-PSP PM, as shown in Figure 4. Summing the DOFs of each parallel module obtains the DOFs of the HS-PM, which is 6.

The three platforms in the 3-PSP- $\underline{P}\left(\mathrm{P}_{\mathrm{H}}\right)$ HS-PM are named base platform, moving platform, and target platform which lie on the fixed plane $\tau$, moving plane $\rho$, and target plane $\varepsilon$, respectively. The 1 st prismatic joint $\left(\mathrm{P}_{1 i}\right.$ with the actuate parameter $\left.d_{1 i}, i=1,2,3\right)$ and the 3rd prismatic joint $\left(\mathrm{P}_{3 i}\right.$, with the actuate parameter $\left.d_{3 i}, i=1,2,3\right)$ are active joints with their translational axes $A_{i} B_{i}$ and $C_{i} D_{i}$ perpendicular to the base platform and the moving platform, respectively. The 2 nd prismatic joint $\left(\mathrm{P}_{2 i}, i=1,2,3\right)$ and the spherical joint $\left(\mathrm{S}_{i}, i=1,2,3\right)$ as passive joints coincide with the plane $\rho$. The higher joint $\left(\mathrm{P}_{\mathrm{H} i}, i=1,2,3\right)$ has 5 DOFs related to the plane $\varepsilon$, as its apex $D_{i}$ lies on the plane $\varepsilon$.

2.2. The Physical Model of the IHPRM. The 3-PSP- $\underline{\mathrm{P}}\left(\mathrm{P}_{\mathrm{H}}\right)$ HS$\mathrm{PM}$ is applied in the IHPRM, with the main frame, immersion hood, and wafer corresponding to the base platform, moving platform, and target platform, respectively, as shown in Figure 5. Three laser displacement sensors (SI-F01, Keyence) are installed on the IH's lower surface, which detect the distance between the emission point and the reflection point, respectively, by emitting and receiving the laser. The three laser displacement sensors are applied to measure the pose of the IH's lower surface with respect to the wafer surface, which integrate the behavior of both the 3 rd prismatic joint and the higher joint $\left(\mathrm{P}_{\mathrm{H}}\right)$. 


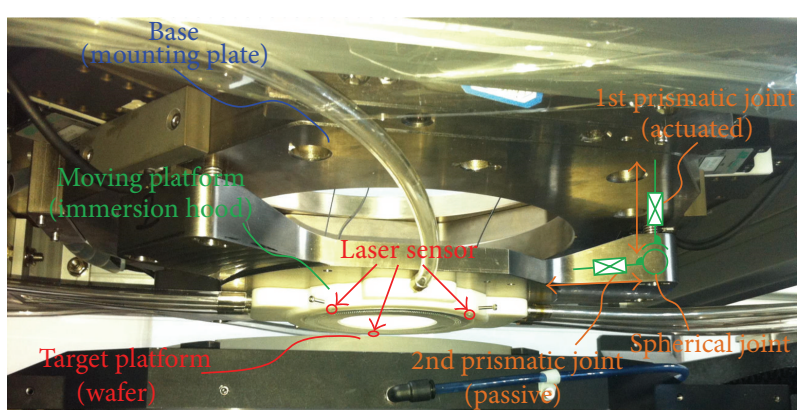

FIGURE 5: The IHPRM in the immersion lithography system.

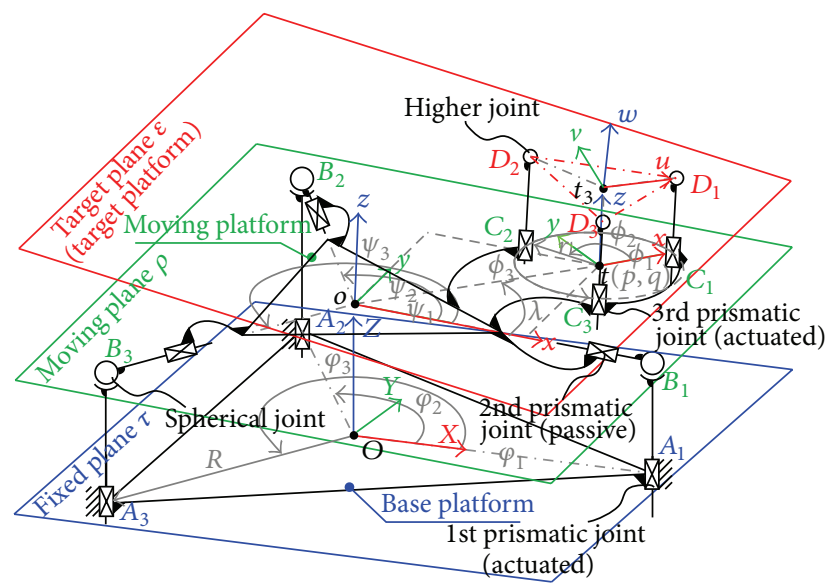

FIGURE 6: The hybrid serial-parallel mechanism with the three reference frames.

\section{Description of Reference Frames and Motion Parameters}

To illustrate the relationship between different components more intuitively, three reference frames are defined in the three reference planes, respectively, as shown in Figure 6. Point $A_{i}(i=1,2,3)$ distributes on a fixed circle with radius of $R$ in the plane $\tau$. A fixed reference frame $O\{X, Y, Z\}(\Sigma O)$ is assigned at the central point $O$ of the fixed circle, with the $O Z$-axis perpendicular to the base platform and the $O X$-axis passing through point $A_{1}$. The angle between $\overrightarrow{\mathbf{O A}_{i}}$ and $O X-$ axis is $\varphi_{i}$. According to the Cartesian right-hand rule, the $O Y$ axis can be determined. The axes of the 2 nd prismatic joints intersect at point $o$. The point $o$ is defined as the center of the platform. Then a moving reference frame $o\{x, y, z\}(\Sigma o)$ is assigned in the plane $\rho$ at point $o$, with $o z$-axis perpendicular to plane $\rho$ and the $o x$-axis passing through point $B_{1}$. The $o y$ axis is also determined by the Cartesian right-hand rule. The angle between $\overrightarrow{\mathbf{o B}}_{i}$ and $o x$-axis is $\psi_{i}$. Point $C_{i}(i=1,2,3)$ distributes on a mobile circle which centered at point $t(p, q)$ with radius of $r$ in the plane $\rho$. Points $o, t$, and $C_{1}$ are collinear, and $\angle B_{1} o t$ is $\lambda$. Another moving reference frame $t\{x, y, z\}\left(\sum t\right)$ is assigned in the plane $\rho$ at point $t$, with $t z$ axis perpendicular to plane $\rho$, and the $t x$-axis passing through point $C_{1}$. The $t y$-axis is also determined by the Cartesian

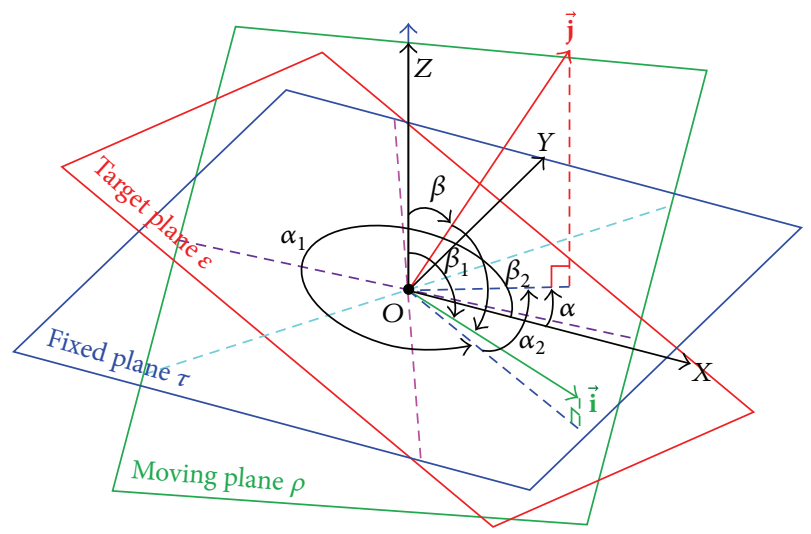

FIGURE 7: The angular relationships between the three planes.

right-hand rule. The angle between $\overrightarrow{\mathbf{t C}}_{i}$ and $t x$-axis is $\phi_{i}$. Point $t_{3}$ is the intersection of the $t z$-axis and the plane $\varepsilon$. A target reference frame $t_{3}\{u, v, w\}\left(\Sigma t_{3}\right)$ is assigned in the plane $\varepsilon$ at point $t_{3}$, with $t_{3} w$-axis perpendicular to plane $\varepsilon$ and the $t_{3} u$ axis passing through the apex $D_{1}$ of the higher joint $\left(\mathrm{P}_{\mathrm{H}}\right)$. The $t_{3} v$-axis is also determined by the Cartesian right-hand rule.

Three parameters are required to describe the pose of the plane $\tau$ and plane $\rho$, respectively, in frame $\Sigma O$, which includes the angular parameters and the position parameters.

3.1. Angular Parameters Definition. The fixed plane $\tau$, moving plane $\rho$, and target plane $\varepsilon$ are moved together and intersect at point $O$, which will not change the angular relationship between them, as shown in Figure 7.

Vector $\overrightarrow{\mathbf{j}}$ is the normal unit vector of the target plane $\varepsilon$; vector $\overrightarrow{\mathbf{i}}$ is the normal unit vector of the moving plane $\rho$. The angle between $\overrightarrow{\mathbf{j}}$ and the $O Z$-axis is defined as tilt angle $\beta$. The angle between $\overrightarrow{\mathbf{j}}$ 's projection on the fixed plane $\tau$ and the $O X$ axis is defined as twist angle $\alpha$. Similarly, the angle between $\overrightarrow{\mathbf{i}}$ and the $O Z$-axis is defined as tilt angle $\beta_{1}$. The angle between $\overrightarrow{\mathrm{i}}$ 's projection on the fixed plane $\tau$ and the $O X$-axis is defined as twist angle $\alpha_{1}$. The angle between the vector $\overrightarrow{\mathbf{j}}$ and vector $\overrightarrow{\mathbf{i}}$ is defined as tilt angle $\beta_{2}$. The angle between $\overrightarrow{\mathbf{j}}$ 's projection and $\overrightarrow{\mathbf{i}}$ 's projection on the fixed plane $\tau$ is defined as twist angle $\alpha_{2}$. As defined above, these six angular parameters have the constraints as follows:

$$
\begin{gathered}
\alpha_{2}=\alpha-\alpha_{1} \\
\cos \beta_{1} \cos \beta+\sin \beta_{1} \sin \beta \cos \alpha_{2}=\cos \beta_{2} .
\end{gathered}
$$

3.2. Position Parameters Definition. As the angular relationships of the fixed plane $\tau$, moving plane $\rho$, and target plane $\varepsilon$ are specified, the position relationships between them should also be determined in frame $\Sigma O$.

As shown in Figure 8, the $O Z$-axis intersects the plane $\varepsilon$ at point $t_{1}$; then the distance between point $O$ and point $t_{1}$ is defined as the central distance $H$ between the fixed plane $\tau$ and the target plane $\varepsilon$. Similarly, point $g_{1}$ is the projection of the moving platform's central point $o$ in the plane $\tau$. The distance between point $o$ and point $g_{1}$ is defined as the central 


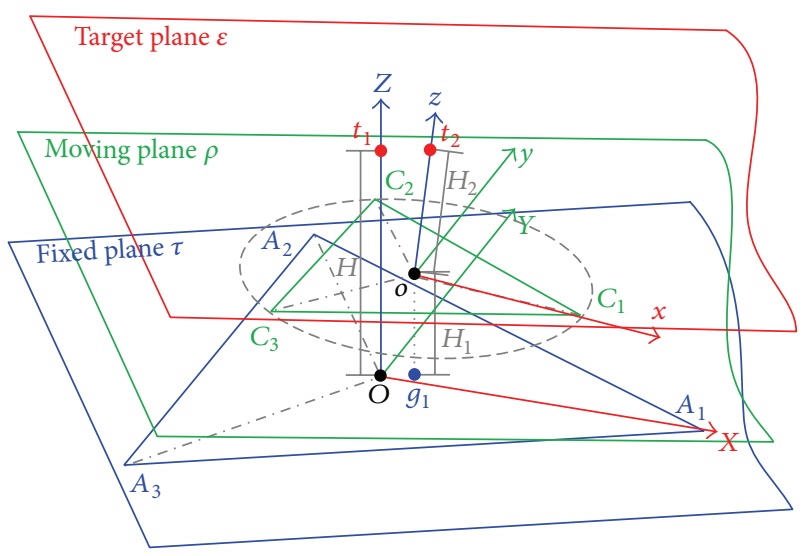

Figure 8: The position relationships between the three planes.

distance $H_{1}$ between the fixed plane $\tau$ and the moving plane $\rho$. Further, $o z$-axis intersects the target plane $\varepsilon$ at point $t_{2}$; the distance between point $o$ and point $t_{2}$ is defined as the central distance $\mathrm{H}_{2}$ between the moving plane $\rho$ and the target plane $\varepsilon$.

The two out of six angular parameters can be obtained by the other arbitrarily specified four parameters, as shown in (2). Similarly, while the two out of three position parameters are specified, the other one position parameter can be calculated, as shown in (47) in Section 5. As a consequence, six couple mixed-type pose parameters (including 4 independent angular parameters and 2 independent position parameters) are required to fully describe the pose relationship between the three planes. The pose description mode based on the couple mixed-type is more significant to the pose control algorithm compared to the nonpure translational mode.

\section{Position Analysis of the 3-PSP Mechanism}

The position analysis for mechanism is comprised of two parts: the direct position analysis and the inverse position analysis. The direct position analysis is to calculate the pose parameters of the platform relative to the base with the given actuated joint parameters, while the inverse position analysis aims to calculate the actuated joint parameters from the given pose of the platform relative to the base [17]. The inverse kinematic analysis of the most parallel mechanism is quite simple and usually has solutions in analytical form, while the direct kinematic analysis is extremely complicated, and only numerical or closed-form solutions can be obtained. As the basis of the 3-PSP- $\underline{\mathrm{P}}\left(\mathrm{P}_{\mathrm{H}}\right)$ HS-PM's position analysis, the position analysis of the 3-PSP mechanism is investigated firstly.

4.1. Direct Position Analysis of the 3-PSP Mechanism. The architecture of the 3-PSP PM and the reference frames are shown in Figures 3 and 6 . The direct position analysis of the 3-PSP PM aims to obtain the orientation parameters $\left(\alpha_{1}\right.$, $\left.\beta_{1}\right)$ and position parameter $\left(H_{1}\right)$ of the moving platform with respect to the base platform using the given actuated parameters $d_{1 i}(i=1,2,3)$ of the actuate prismatic joints $\mathrm{P}_{1 i}$.
4.1.1. Tilt Angle $\beta_{1}$. The homogeneous coordinates of $A_{i}$ and $B_{i}$ in the fixed reference frame $\Sigma O$ are

$$
\begin{gathered}
\mathbf{A}_{i \sum O}=\left[\begin{array}{llll}
R \cos \varphi_{i} & R \sin \varphi_{i} & 0 & 1
\end{array}\right]^{T} \quad(i=1,2,3) \\
\mathbf{B}_{i \sum O}=\left[\begin{array}{llll}
R \cos \varphi_{i} & R \sin \varphi_{i} & d_{1 i} & 1
\end{array}\right]^{T} \quad(i=1,2,3),
\end{gathered}
$$

where the additional right side subscripts of $A_{i}$ and $B_{i}$ indicate the reference frame in which the points are measured.

Since the spherical joint $S_{i}$ 's central point $B_{i}(i=1,2,3)$ is in the moving plane $\rho$, the normal vector of the plane $\rho$ can

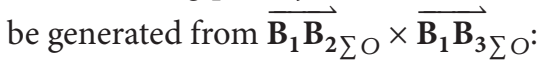

$$
\begin{aligned}
& \overrightarrow{\mathbf{B}_{1} \mathbf{B}_{2 \sum O}} \\
& =\left[\begin{array}{llll}
R\left(\cos \varphi_{2}-\cos \varphi_{1}\right) & R\left(\sin \varphi_{2}-\sin \varphi_{1}\right) & d_{12}-d_{11} & 0
\end{array}\right]^{T} \\
& \overrightarrow{\mathbf{B}_{1} \mathbf{B}_{3 \sum O}} \\
& =\left[\begin{array}{lll}
R\left(\cos \varphi_{3}-\cos \varphi_{1}\right) R\left(\sin \varphi_{3}-\sin \varphi_{1}\right) & d_{13}-d_{11} & 0
\end{array}\right]^{T} .
\end{aligned}
$$

Then we have the normal unit vector $\overrightarrow{\mathbf{k}}_{1 \sum \mathrm{O}}$ of the plane $\rho$ as follows:

$$
\begin{aligned}
\vec{k}_{1 \sum O} & =\frac{\overrightarrow{\mathbf{B}_{1} \mathbf{B}_{2 \sum O}} \times \overrightarrow{\mathbf{B}_{1} \mathbf{B}_{3 \sum O}}}{\left|\overrightarrow{\mathbf{B}_{1} \mathbf{B}_{2 \sum O}} \times \overrightarrow{\mathbf{B}_{1} \mathbf{B}_{3 \sum O}}\right|} \\
& =\frac{1}{\sqrt{k_{11}^{2}+k_{12}^{2}+k_{13}^{2}}}\left[\begin{array}{llll}
k_{11} & k_{12} & k_{13} & 0
\end{array}\right]^{T},
\end{aligned}
$$

where

$$
\begin{aligned}
k_{11}= & \left(d_{12}-d_{13}\right) \sin \varphi_{1}+\left(d_{13}-d_{11}\right) \sin \varphi_{2} \\
& +\left(d_{11}-d_{12}\right) \sin \varphi_{3}, \\
k_{12}= & \left(d_{13}-d_{12}\right) \cos \varphi_{1}+\left(d_{11}-d_{13}\right) \cos \varphi_{2} \\
& +\left(d_{12}-d_{11}\right) \cos \varphi_{3}, \\
k_{13}= & R\left[\sin \left(\varphi_{1}-\varphi_{3}\right)+\sin \left(\varphi_{2}-\varphi_{1}\right)+\sin \left(\varphi_{3}-\varphi_{2}\right)\right] .
\end{aligned}
$$

The equation of the moving plane $\rho$ in the frame $\Sigma O$ is

$$
k_{11}\left(x-R \cos \varphi_{1}\right)+k_{12}\left(y-R \sin \varphi_{1}\right)+k_{13}\left(z-d_{11}\right)=0 .
$$

As defined above, the tilt angle $\beta_{1}$, from the normal unit vector $\overrightarrow{\mathbf{k}}_{1 \sum O}$ to the positive direction of $O Z$-axis, is limited within the range of $[0, \pi / 2)$, which can be expressed as

$$
\beta_{1}=\arctan \left(\frac{\sqrt{k_{11}^{2}+k_{12}^{2}}}{k_{13}}\right) .
$$


4.1.2. Twist Angle $\alpha_{1}$. Let the unit vector $\overrightarrow{\mathbf{w}}_{1 \sum \mathrm{O}}$ denote the direction of the normal unit vector $\overrightarrow{\mathbf{k}}_{1 \sum O}$ 's projection on the fixed plane $\tau$

$$
\overrightarrow{\mathbf{w}}_{1 \sum O}=\frac{1}{\sqrt{k_{11}^{2}+k_{12}^{2}}}\left[\begin{array}{llll}
k_{11} & k_{12} & 0 & 0
\end{array}\right]^{T} .
$$

Calculating the angle between vector $\overrightarrow{\mathbf{w}}_{1 \sum \mathrm{O}}$ and the positive $O X$-axis yields the twist angle $\alpha_{1}$, within the range of $(-\pi, \pi]$ as defined above, which can be expressed as

$$
\alpha_{1}=\operatorname{atan} 2\left(k_{12}, k_{11}\right)
$$

where atan 2 function calculates the arc tangent of the two variables $k_{12}$ and $k_{11}$, in which the signs of both arguments are used to determine the quadrant of the result.

4.1.3. Central Distance $H_{1}$. The unit vector which denotes the direction of the prismatic joint $P_{2 i}$ 's axis in the frame $\Sigma o$ is

$$
\overrightarrow{\mathbf{n}_{2 i \sum o}}=\left[\begin{array}{llll}
\cos \psi_{i} & \sin \psi_{i} & 0 & 0
\end{array}\right]^{T} \quad(i=1,2,3) .
$$

The homogeneous transformation matrices $\mathbf{T}_{o}^{O}$ which transfers the coordinates in the frame $\Sigma o$ to the coordinates in the frame $\Sigma O$ can be obtained by the Euler's transformation method:

$$
\begin{gathered}
\mathbf{T}_{o}^{O}=\left[\begin{array}{ll}
\mathbf{R} & \mathbf{P} \\
\mathbf{0} & \mathbf{1}
\end{array}\right] \\
\mathbf{R}=\operatorname{Rot}\left(z, \alpha_{1}\right) \cdot \operatorname{Rot}\left(y, \beta_{1}\right) \cdot \operatorname{Rot}\left(z, \gamma_{1}\right) \\
=\left[\begin{array}{lll}
R_{11} & R_{12} & R_{13} \\
R_{21} & R_{22} & R_{23} \\
R_{31} & R_{32} & R_{33}
\end{array}\right],
\end{gathered}
$$

where $\mathbf{P}$ denotes vector from the point $O$ to the point $o$ in the fixed reference frame $\Sigma O . \mathbf{R}$ is the Euler matrix. $\boldsymbol{\operatorname { R o t }}\left(z, \alpha_{1}\right)$ denotes the counterclockwise rotation angle $\alpha_{1}$ about the $o z$-axis, $\boldsymbol{\operatorname { R o t }}\left(y, \beta_{1}\right)$ indicates the counterclockwise rotation angle $\beta_{1}$ about the $o y$-axis, and $\operatorname{Rot}\left(z, \gamma_{1}\right)$ indicates the counterclockwise rotation angle $\gamma_{1}$ about the oz-axis. Consider the following:

$$
\begin{aligned}
& R_{11}=\cos \alpha_{1} \cos \beta_{1} \cos \gamma_{1}-\sin \alpha_{1} \sin \gamma_{1} ; \\
& R_{12}=-\cos \alpha_{1} \cos \beta_{1} \sin \gamma_{1}-\sin \alpha_{1} \cos \gamma_{1} ; \\
& R_{13}=\cos \alpha_{1} \sin \beta_{1} ; \\
& R_{21}=\sin \alpha_{1} \cos \beta_{1} \cos \gamma_{1}+\cos \alpha_{1} \sin \gamma_{1} ; \\
& R_{22}=-\sin \alpha_{1} \cos \beta_{1} \sin \gamma_{1}+\cos \alpha_{1} \cos \gamma_{1} ; \\
& R_{23}=\sin \alpha_{1} \sin \beta_{1} ; \\
& R_{31}=-\sin \beta_{1} \cos \gamma_{1} ; \\
& R_{32}=\sin \beta_{1} \sin \gamma_{1} ; \\
& R_{33}=\cos \beta_{1} .
\end{aligned}
$$

Assume the coordinate of the moving platform's central point $o$ in the frame $\Sigma O$ as

$$
\mathbf{o}_{\sum O}=\left[\begin{array}{llll}
o_{x} & o_{y} & o_{z} & 1
\end{array}\right]^{T} .
$$

The coordinates of the vectors from point $o$ to point $A_{i}$ are

$$
\begin{aligned}
& \overrightarrow{\mathbf{o A}_{\mathbf{i} \sum O}}=\left[\begin{array}{llll}
R \cos \varphi_{i}-o_{x} & R \sin \varphi_{i}-o_{y} & -o_{z} & 0
\end{array}\right]^{T} \\
& (i=1,2,3) \text {. }
\end{aligned}
$$

The unit vector of the $O Z$-axis in the frame $\Sigma O$ is

$$
\overrightarrow{\boldsymbol{\tau}}_{\sum O}=\left[\begin{array}{llll}
0 & 0 & 1 & 0
\end{array}\right]^{T}
$$

As the vectors $\overrightarrow{\mathbf{n}}_{\mathbf{i} \sum O}, \overrightarrow{\mathbf{o} \mathbf{A}_{\mathbf{i}}}$, and $\overrightarrow{\boldsymbol{\tau}}_{\sum O}$ are coplanar according to the geometrical constraint $[19,24]$, their mixed product equals zero:

$$
\left\|\overrightarrow{\mathbf{n}}_{\mathbf{i} \sum O} \overrightarrow{\mathbf{o A}}_{\mathbf{i} \sum O} \overrightarrow{\boldsymbol{\tau}}_{\sum O}\right\|=0 \quad(i=1,2,3) .
$$

Equation (16) can be rearranged to obtain three constraint equations:

$$
\begin{array}{r}
\left(R_{21} o_{x}-R_{11} o_{y}\right) \cos \psi_{i}+\left(R_{22} o_{x}-R_{12} o_{y}\right) \sin \psi_{i} \\
+R \cdot R_{11} \cos \psi_{i} \sin \varphi_{i}-R \cdot R_{22} \cos \varphi_{i} \sin \psi_{i} \\
+R \cdot R_{12} \sin \varphi_{i} \sin \psi_{i}-R \cdot R_{21} \cos \varphi_{i} \cos \psi_{i}=0 \\
(i=1,2,3) .
\end{array}
$$

The three constraint equations yield the constraint equation of the torsion angle $\gamma_{1}$

$$
a R_{11}+b R_{12}+c R_{21}+d R_{22}=0
$$

where

$$
\begin{aligned}
a= & \sin \psi_{1} \cos \psi_{2} \cos \psi_{3} \sin \varphi_{3}-\sin \psi_{1} \cos \psi_{2} \cos \psi_{3} \sin \varphi_{2} \\
& +\cos \psi_{1} \sin \psi_{2} \cos \psi_{3} \sin \varphi_{1} \\
& -\cos \psi_{1} \sin \psi_{2} \cos \psi_{3} \sin \varphi_{3} \\
& -\cos \psi_{1} \cos \psi_{2} \sin \psi_{3} \sin \varphi_{1} \\
& +\cos \psi_{1} \cos \psi_{2} \sin \psi_{3} \sin \varphi_{2} \\
b= & \sin \psi_{1} \sin \psi_{2} \cos \psi_{3} \sin \varphi_{1}-\sin \psi_{1} \sin \psi_{2} \cos \psi_{3} \sin \varphi_{2} \\
& -\sin \psi_{1} \cos \psi_{2} \sin \psi_{3} \sin \varphi_{1}
\end{aligned}
$$




$$
\begin{aligned}
& +\sin \psi_{1} \cos \psi_{2} \sin \psi_{3} \sin \varphi_{3} \\
& +\cos \psi_{1} \sin \psi_{2} \sin \psi_{3} \sin \varphi_{2} \\
& -\cos \psi_{1} \sin \psi_{2} \sin \psi_{3} \sin \varphi_{3} ; \\
c= & \cos \psi_{2} \sin \left(\psi_{1}-\psi_{3}\right) \cos \varphi_{2}-\cos \psi_{3} \sin \left(\psi_{1}-\psi_{2}\right) \cos \varphi_{3} \\
& -\cos \psi_{1} \sin \psi_{2} \cos \psi_{3} \cos \varphi_{1} \\
& +\cos \psi_{1} \cos \psi_{2} \sin \psi_{3} \cos \varphi_{1} ; \\
d= & \sin \psi_{2} \sin \left(\psi_{1}-\psi_{3}\right) \cos \varphi_{2}-\sin \psi_{1} \sin \psi_{2} \cos \psi_{3} \cos \varphi_{1} \\
& +\sin \psi_{1} \sin \psi_{3} \cos \psi_{2} \cos \varphi_{1} \\
& -\sin \psi_{3} \sin \left(\psi_{1}-\psi_{2}\right) \cos \varphi_{3} ;
\end{aligned}
$$

when $\cos \gamma_{1}=0$,

$$
\gamma_{1}= \begin{cases}-\frac{\alpha_{1}}{\left|\alpha_{1}\right|} \frac{\pi}{2} & \alpha_{1} \neq 0 \\ \frac{\pi}{2} & \alpha_{1}=0\end{cases}
$$

The coordinates $o_{x}$ and $o_{y}$ of the central point $o$ in the frame $\Sigma O$ can be expressed in the analytical form

$$
\begin{aligned}
& o_{x}=\left(-R \cos \varphi_{1}\left(R_{21} \cos \psi_{1}+R_{22} \sin \psi_{1}\right)\right. \\
& \left.\cdot\left(R_{11} \cos \psi_{2}+R_{12} \sin \psi_{2}\right)\right) \\
& \cdot\left(\left(R_{12} R_{21}-R_{11} R_{22}\right) \sin \left(\psi_{1}-\psi_{2}\right)\right)^{-1} \\
& +\left(R\left(R_{11} \cos \psi_{1}+R_{12} \sin \psi_{1}\right)\left(\sin \varphi_{1}-\sin \varphi_{2}\right)\right. \\
& \left.\cdot\left(R_{11} \cos \psi_{2}+R_{12} \sin \psi_{2}\right)\right) \\
& \cdot\left(\left(R_{12} R_{21}-R_{11} R_{22}\right) \sin \left(\psi_{1}-\psi_{2}\right)\right)^{-1} \\
& +\left(R\left(R_{11} \cos \psi_{1}+R_{12} \sin \psi_{1}\right) \cos \varphi_{2}\right. \\
& \left.\cdot\left(R_{21} \cos \psi_{2}+R_{22} \sin \psi_{2}\right)\right) \\
& \cdot\left(\left(R_{12} R_{21}-R_{11} R_{22}\right) \sin \left(\psi_{1}-\psi_{2}\right)\right)^{-1} \text {, } \\
& o_{y}=\left(R \sin \varphi_{1}\left(R_{11} \cos \psi_{1}+R_{12} \sin \psi_{1}\right)\right. \\
& \left.\cdot\left(R_{21} \cos \psi_{2}+R_{22} \sin \psi_{2}\right)\right) \\
& \cdot\left(\left(R_{12} R_{21}-R_{11} R_{22}\right) \sin \left(\psi_{1}-\psi_{2}\right)\right)^{-1} \\
& -\left(R \sin \varphi_{2}\left(R_{11} \cos \psi_{2}+R_{12} \sin \psi_{2}\right)\right. \\
& \left.\cdot\left(R_{21} \cos \psi_{1}+R_{22} \sin \psi_{1}\right)\right) \\
& \cdot\left(\left(R_{12} R_{21}-R_{11} R_{22}\right) \sin \left(\psi_{1}-\psi_{2}\right)\right)^{-1} \\
& +\left(R\left(\cos \varphi_{2}-\cos \varphi_{1}\right)\left(R_{21} \cos \psi_{1}+R_{22} \sin \psi_{1}\right)\right. \\
& \left.\cdot\left(R_{21} \cos \psi_{2}+R_{22} \sin \psi_{2}\right)\right) \\
& \cdot\left(\left(R_{12} R_{21}-R_{11} R_{22}\right) \sin \left(\psi_{1}-\psi_{2}\right)\right)^{-1} \text {; }
\end{aligned}
$$

when $\cos \gamma_{1} \neq 0, K_{11}=R_{11} / \cos \gamma_{1}=\cos \alpha_{1} \cos \beta_{1}-$ $\sin \alpha_{1} \tan \gamma_{1} ; K_{12}=R_{12} / \cos \gamma_{1}=-\cos \alpha_{1} \cos \beta_{1} \tan \gamma_{1}-$ $\sin \alpha_{1} ; K_{21}=R_{21} / \cos \gamma_{1}=\sin \alpha_{1} \cos \beta_{1}+\cos \alpha_{1} \tan \gamma_{1}$; $K_{22}=R_{22} / \cos \gamma_{1}=-\sin \alpha_{1} \cos \beta_{1} \tan \gamma_{1}+\cos \alpha_{1}$.

Equation (18) can be transformed as

$$
a K_{11}+b K_{12}+c K_{21}+d K_{22}=0 .
$$

The tangent of the torsion angle $\gamma_{1}$ is

$\tan \gamma_{1}$

$$
=\frac{a \cos \alpha_{1} \cos \beta_{1}-b \sin \alpha_{1}+c \sin \alpha_{1} \cos \beta_{1}+d \cos \alpha_{1}}{a \sin \alpha_{1}+b \cos \beta_{1} \cos \alpha_{1}-c \cos \alpha_{1}+d \sin \alpha_{1} \cos \beta_{1}} .
$$

Then the $o_{x}$ and $o_{y}$ of the central point $o$ in the frame $\Sigma O$ are

$$
\begin{aligned}
& o_{x}=\left(-R \cos \varphi_{1}\left(K_{21} \cos \psi_{1}+K_{22} \sin \psi_{1}\right)\right. \\
& \left.\cdot\left(K_{11} \cos \psi_{2}+K_{12} \sin \psi_{2}\right)\right) \\
& \cdot\left(\left(K_{12} K_{21}-K_{11} K_{22}\right) \sin \left(\psi_{1}-\psi_{2}\right)\right)^{-1} \\
& +\left(R\left(K_{11} \cos \psi_{1}+K_{12} \sin \psi_{1}\right)\left(\sin \varphi_{1}-\sin \varphi_{2}\right)\right. \\
& \left.\cdot\left(K_{11} \cos \psi_{2}+K_{12} \sin \psi_{2}\right)\right) \\
& \cdot\left(\left(K_{12} K_{21}-K_{11} K_{22}\right) \sin \left(\psi_{1}-\psi_{2}\right)\right)^{-1} \\
& +\left(R\left(K_{11} \cos \psi_{1}+K_{12} \sin \psi_{1}\right) \cos \varphi_{2}\right. \\
& \left.\cdot\left(K_{21} \cos \psi_{2}+K_{22} \sin \psi_{2}\right)\right) \\
& \cdot\left(\left(K_{12} K_{21}-K_{11} K_{22}\right) \sin \left(\psi_{1}-\psi_{2}\right)\right)^{-1} \text {, } \\
& o_{y}=\left(R \sin \varphi_{1}\left(K_{11} \cos \psi_{1}+K_{12} \sin \psi_{1}\right)\right. \\
& \left.\cdot\left(K_{21} \cos \psi_{2}+K_{22} \sin \psi_{2}\right)\right) \\
& \cdot\left(\left(K_{12} K_{21}-K_{11} K_{22}\right) \sin \left(\psi_{1}-\psi_{2}\right)\right)^{-1} \\
& -\left(R \sin \varphi_{2}\left(K_{11} \cos \psi_{2}+K_{12} \sin \psi_{2}\right)\right. \\
& \left.\cdot\left(K_{21} \cos \psi_{1}+K_{22} \sin \psi_{1}\right)\right) \\
& \cdot\left(\left(K_{12} K_{21}-K_{11} K_{22}\right) \sin \left(\psi_{1}-\psi_{2}\right)\right)^{-1} \\
& +\left(R\left(\cos \varphi_{2}-\cos \varphi_{1}\right)\left(K_{21} \cos \psi_{1}+K_{22} \sin \psi_{1}\right)\right. \\
& \left.\cdot\left(K_{21} \cos \psi_{2}+K_{22} \sin \psi_{2}\right)\right) \\
& \cdot\left(\left(K_{12} K_{21}-K_{11} K_{22}\right) \sin \left(\psi_{1}-\psi_{2}\right)\right)^{-1} \text {. }
\end{aligned}
$$

As the moving platform's central point $o$ is in plane $\rho$, the coordinate value $o_{z}$ in the $O Z$ direction can be obtained by (7). The distance between the point $o$ and the fixed plane $\tau$ is the central distance $H_{1}$ as defined before

$$
H_{1}=o_{z}=d_{11}-\frac{k_{11}\left(o_{x}-R \cos \varphi_{1}\right)+k_{12}\left(o_{y}-R \sin \varphi_{1}\right)}{k_{13}} .
$$


4.2. Inverse Position Analysis of the 3-PSP Mechanism. The inverse position analysis of the 3-PSP PM is to obtain the actuated parameters $d_{1 i}(i=1,2,3)$ of the prismatic joints $\mathrm{P}_{1 i}$ with the given orientation parameters $\left(\alpha_{1}, \beta_{1}\right)$ and position parameter $\left(H_{1}\right)$ of the moving platform relative to the base platform.

The coordinate values $o_{x}$ and $o_{y}$ in the frame $\Sigma O$ can be obtained directly according to the direct position analysis in Section 4.1. The homogeneous coordinate of the moving platform's central point $o$ is

$$
\mathbf{o}_{\sum O}=\left[\begin{array}{llll}
o_{x} & o_{y} & o_{z} & 1
\end{array}\right]^{T},
$$

where $o_{z}=H_{1}$.

The vector $\overrightarrow{\mathbf{o B}}_{\mathbf{i}}$ indicates the direction from the moving platform's central point $o$ to the spherical joint's central point $B_{i}$ in the plane $\rho$

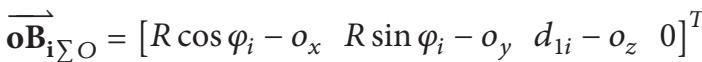

$$
\begin{aligned}
& (i=1,2,3) \text {. }
\end{aligned}
$$

The normal unit vector $\overrightarrow{\mathbf{k}}_{1 \sum o}=\left[\begin{array}{llll}0 & 0 & 1 & 0\end{array}\right]^{T}$ of the plane $\rho$ in the frame $\Sigma o$ can be transferred to $\overrightarrow{\mathbf{k}}_{1 \sum O}$ in the frame $\Sigma O$ with the given twist angle $\alpha_{1}$ and tilt angle $\beta_{1}$ :

$$
\begin{gathered}
\overrightarrow{\mathbf{k}}_{1 \sum O}=\left[\begin{array}{ll}
\mathbf{R} & \mathbf{0} \\
\mathbf{0} & \mathbf{1}
\end{array}\right] \cdot \overrightarrow{\mathbf{k}}_{1 \sum o} \\
\mathbf{R}=\operatorname{Rot}\left(z, \alpha_{1}\right) \mathbf{R o t}\left(y, \beta_{1}\right) \\
=\left[\begin{array}{ccc}
\cos \alpha_{1} \cos \beta_{1} & -\sin \alpha_{1} & \cos \alpha_{1} \sin \beta_{1} \\
\sin \alpha_{1} \cos \beta_{1} & \cos \alpha_{1} & \sin \alpha_{1} \sin \beta_{1} \\
-\sin \beta_{1} & 0 & \cos \beta_{1}
\end{array}\right],
\end{gathered}
$$

where $\mathbf{R}$ is the transformation matric. $\operatorname{Rot}\left(z, \alpha_{1}\right)$ denotes the counterclockwise rotation angle $\alpha_{1}$ about the oz-axis; $\boldsymbol{\operatorname { R o t }}\left(y, \beta_{1}\right)$ indicates the counterclockwise rotation angle $\beta_{1}$ about the $o y$-axis. Consider

$$
\overrightarrow{\mathbf{k}}_{1 \sum O}=\left[\begin{array}{llll}
\cos \alpha_{1} \sin \beta_{1} & \sin \alpha_{1} \sin \beta_{1} & \cos \beta_{1} & 0
\end{array}\right]^{T} .
$$

Since the vector $\overrightarrow{\mathbf{o B}}_{\mathbf{i} \sum O}$ in the plane $\rho$ is orthogonal with respect to the normal unit vector $\overrightarrow{\mathbf{k}}_{1 \sum \mathrm{O}}$ of the plane $\rho$, the following equation can be obtained:

$$
\begin{gathered}
\overrightarrow{\mathbf{o B}}_{\mathbf{i} \sum O} \cdot \overrightarrow{\mathbf{k}}_{1 \sum O}=0 \quad(i=1,2,3) \\
d_{1 i}=o_{z}-\left[\left(R \cos \varphi_{i}-o_{x}\right) \cos \alpha_{1}\right. \\
\left.+\left(R \sin \varphi_{i}-o_{y}\right) \sin \alpha_{1}\right] \tan \beta_{1} .
\end{gathered}
$$

Overall, by choosing the proper pose parameters and constraints, both the direct and inverse position analyses of the 3-PSP PM obtain the solutions in analytical form, which indicates the one-to-one correspondent relationships between the actuated parameters and the pose parameters of the 3-PSP PM.

\section{Position Analysis of the Hybrid Serial-Parallel Mechanism}

5.1. Direct Position Analysis of the Hybrid Serial-Parallel Mechanism. The direct position analysis of the 3-PSP-P $\left(\mathrm{P}_{\mathrm{H}}\right) \mathrm{HS}$ $\mathrm{PM}$ aims to obtain the pose parameters of the target plane $\varepsilon$ relative to the fixed plane $\tau(\alpha, \beta, H)$ and the moving plane $\rho$ $\left(\alpha_{2}, \beta_{2}, H_{2}\right)$, respectively, using the given actuated parameters $d_{1 i}$ and $d_{3 i}(i=1,2,3)$ of the prismatic joints $\mathrm{P}_{1 i}$ and $\mathrm{P}_{3 i}$.

As the actuated parameters $d_{1 i}(i=1,2,3)$ are given, the parameters $\alpha_{1}, \beta_{1}$, and $H_{1}$ which describe the pose of the plane $\rho$ with respect to the plane $\tau$ can be obtained directly as the direct position analysis of the 3-PSP PM shown in Section 4.1 .

The actuated parameters $d_{3 i}(i=1,2,3)$ of the prismatic joints equal the module of the vector $\overrightarrow{\mathbf{C}_{\mathbf{i}} \mathbf{D}_{\mathbf{i}}}$. As shown in the Section 4 , the normal unit vector $\overrightarrow{\mathbf{k}}_{2 \sum o}$ of the target plane $\varepsilon$ in frame $\Sigma t$ is

$$
\overrightarrow{\mathbf{k}}_{2 \sum t}=\frac{1}{\sqrt{k_{21}^{\prime \prime 2}+k_{22}^{\prime \prime 2}+k_{23}^{\prime \prime 2}}}\left[\begin{array}{llll}
k_{21}^{\prime \prime} & k_{22}^{\prime \prime} & k_{23}^{\prime \prime} & 0
\end{array}\right]^{T},
$$

where

$$
\begin{aligned}
k_{21}^{\prime \prime}= & \left(d_{32}-d_{33}\right) \sin \phi_{1}+\left(d_{33}-d_{31}\right) \sin \phi_{2} \\
& +\left(d_{31}-d_{32}\right) \sin \phi_{3}, \\
k_{22}^{\prime \prime}= & \left(d_{33}-d_{32}\right) \cos \phi_{1}+\left(d_{31}-d_{33}\right) \cos \phi_{2} \\
& +\left(d_{32}-d_{31}\right) \cos \phi_{3}, \\
k_{23}^{\prime \prime}= & r\left[\sin \left(\phi_{1}-\phi_{3}\right)+\sin \left(\phi_{2}-\phi_{1}\right)+\sin \left(\phi_{3}-\phi_{2}\right)\right] .
\end{aligned}
$$

$\overrightarrow{\mathbf{k}}_{2 \sum t}$ can be transferred to the frame $\Sigma o$, as

$$
\begin{aligned}
\overrightarrow{\mathbf{k}}_{2 \sum o} & =\mathbf{T}_{t}^{o} \cdot \overrightarrow{\mathbf{k}}_{2 \sum t} \\
& =\frac{1}{\sqrt{k_{21}^{\prime 2}+k_{22}^{\prime 2}+k_{23}^{\prime 2}}}\left[\begin{array}{llll}
k_{21}^{\prime} & k_{22}^{\prime} & k_{23}^{\prime} & 0
\end{array}\right]^{T},
\end{aligned}
$$

where

$$
\begin{gathered}
\mathbf{T}_{t}^{o}=\left[\begin{array}{cccc}
\cos \lambda & -\sin \lambda & 0 & p \\
\sin \lambda & \cos \lambda & 0 & q \\
0 & 0 & 1 & 0 \\
0 & 0 & 0 & 1
\end{array}\right], \\
k_{21}^{\prime}=k_{21}^{\prime \prime} \cos \lambda-k_{22}^{\prime \prime} \sin \lambda, \\
k_{22}^{\prime}=k_{21}^{\prime \prime} \sin \lambda+k_{22}^{\prime \prime} \cos \lambda, \\
k_{23}^{\prime}=k_{23}^{\prime \prime} .
\end{gathered}
$$


$\overrightarrow{\mathbf{k}}_{2 \sum o}$ can be transferred to the frame $\Sigma O$, as

$$
\overrightarrow{\mathbf{k}}_{2 \sum O}=\left[\begin{array}{ll}
\mathbf{R} & \mathbf{0} \\
\mathbf{0} & \mathbf{1}
\end{array}\right] \cdot \overrightarrow{\mathbf{k}}_{2 \sum o}=\frac{1}{\sqrt{k_{21}^{2}+k_{22}^{2}+k_{23}^{2}}}\left[\begin{array}{c}
k_{21} \\
k_{22} \\
k_{23} \\
0
\end{array}\right],
$$

where

$$
\begin{aligned}
& k_{21}=k_{21}^{\prime} \cos \alpha_{1} \cos \beta_{1}-k_{22}^{\prime} \sin \alpha_{1}+k_{23}^{\prime} \cos \alpha_{1} \sin \beta_{1}, \\
& k_{22}=k_{21}^{\prime} \sin \alpha_{1} \cos \beta_{1}+k_{22} \cos \alpha_{1}+k_{23}^{\prime} \sin \alpha_{1} \sin \beta_{1}, \\
& k_{23}=-k_{21}^{\prime} \sin \beta_{1}+k_{23}^{\prime} \cos \beta_{1} .
\end{aligned}
$$

As defined above, the tilt angle $\beta$, from the normal unit vector $\overrightarrow{\mathbf{k}}_{2 \sum O}$ to the positive direction of $O Z$-axis, is limited within the range of $[0, \pi / 2)$, which can be expressed as

$$
\beta=\arctan \left(\frac{\sqrt{k_{21}^{2}+k_{22}^{2}}}{k_{23}}\right) \text {. }
$$

Let the unit vector $\overrightarrow{\mathbf{w}}_{2 \sum \mathrm{O}}$ denote the direction of the normal unit vector $\overrightarrow{\mathbf{k}}_{2 \sum O}$ 's projection on the fixed plane $\tau$

$$
\overrightarrow{\mathbf{w}}_{2 \sum O}=\frac{1}{\sqrt{k_{21}^{2}+k_{22}^{2}}}\left[\begin{array}{llll}
k_{21} & k_{22} & 0 & 0
\end{array}\right]^{T} .
$$

Calculating the angle from vector $\overrightarrow{\mathbf{w}}_{2 \sum \mathrm{O}}$ to the positive $O X$-axis yields the twist angle $\alpha$, within the range of $(-\pi, \pi]$ as defined above, which can be expressed as

$$
\alpha=\operatorname{atan} 2\left(k_{22}, k_{21}\right) .
$$

The tilt angle $\beta_{2}$ and twist angle $\alpha_{2}$ can be calculated according to (2).

In order to obtain the central distance $\mathrm{H}_{2}$, an intermediate variable $d_{c}$ is introduced here, which indicates the distance between the points $t$ and $t_{3}$ :

$$
\begin{aligned}
d_{c}= & \left(-2 d_{32} \cos \left(\frac{\phi_{3}}{2}\right)+\csc \left(\frac{\phi_{3}}{2}\right)\right. \\
& \left.\cdot\left[d_{33} \sin \phi_{2}-d_{31} \sin \left(\phi_{2}-\phi_{3}\right)\right]\right) \\
& \cdot\left(2\left[\cos \left(\phi_{2}-\frac{\phi_{3}}{2}\right)-\cos \left(\frac{\phi_{3}}{2}\right)\right]\right)^{-1} .
\end{aligned}
$$

The target plane $\varepsilon$ in the frame $\Sigma t$ is

$$
k_{21}^{\prime \prime}(u-0)+k_{22}^{\prime \prime}(v-0)+k_{23}^{\prime \prime}\left(w-d_{c}\right)=0 .
$$

Transfer the coordinate of point $t_{2}$ in the frame $\Sigma o$ to the frame $\Sigma t$

$$
\begin{aligned}
\mathbf{t}_{2 \sum t} & =\left[\mathbf{T}_{t}^{o}\right]^{-1} \cdot\left[\begin{array}{llll}
0 & 0 & H_{2} & 1
\end{array}\right]^{T} \\
& =\left[\begin{array}{llll}
-p \cos \lambda-q \sin \lambda & -q \cos \lambda+p \sin \lambda & H_{2} & 1
\end{array}\right]^{T} .
\end{aligned}
$$

By substituting formula (42) into (41), $\mathrm{H}_{2}$ can be obtained as follows:

$$
H_{2}=\frac{k_{21}^{\prime \prime}(p \cos \lambda+q \sin \lambda)+k_{22}^{\prime \prime}(q \cos \lambda-p \sin \lambda)}{k_{23}^{\prime \prime}}+d_{c} .
$$

By transfer the coordinate of point $t_{2}$ in frame $\Sigma o$ to frame $\Sigma O$ with the homogeneous transformation matrices $\mathbf{T}_{o}^{O}$

$$
\begin{aligned}
& \mathbf{t}_{2 \sum O}=\left[\begin{array}{llll}
t_{2 x \sum O} & t_{2 y}{ }_{\sum O} & t_{2 z \sum O} & 1
\end{array}\right]^{T} \\
& =\mathbf{T}_{\mathbf{o}}^{\mathbf{O}} \cdot\left[\begin{array}{llll}
0 & 0 & H_{2} & 1
\end{array}\right]^{T} .
\end{aligned}
$$

The equation of the target plane $\varepsilon$ is

$$
k_{21}\left(x-t_{2 x \sum O}\right)+k_{22}\left(y-t_{2 y \sum O}\right)+k_{23}\left(z-t_{2 z \sum O}\right)=0 .
$$

Let $x=0$ and $y=0$ yield the central distance $H$ :

$$
H=z=\frac{k_{21} t_{2 x \sum O}+k_{22} t_{2 y}{ }_{\sum O}}{k_{23}}+t_{2 z \sum O} .
$$

The relationship between the central distances $H, H_{1}$, and $\mathrm{H}_{2}$ can be obtained with (44) and (46):

$$
H_{1}=o_{z}=H-\frac{k_{21} t_{2 x \sum O}+k_{22} t_{2 y} \sum \mathrm{O}}{k_{23}}-H_{2} R_{33} \text {. }
$$

5.2. Inverse Position Analysis of the Hybrid Serial-Parallel Mechanism. The inverse position analysis of the 3-PSP-P $\left(\mathrm{P}_{\mathrm{H}}\right)$ HS-PM is to obtain the actuated parameters $d_{1 i}$ and $d_{3 i}(i=$ $1,2,3)$ of the prismatic joints $\mathrm{P}_{1 i}$ and $\mathrm{P}_{3 i}$ for the given pose parameters of the target plane $\varepsilon$ relative to the fixed plane $\tau$ $(\alpha, \beta, H)$ and the moving plane $\rho\left(\alpha_{2}, \beta_{2}, H_{2}\right)$, respectively.

The homogeneous coordinate of the higher joint's apex $D_{i}$ in frame $\Sigma o$ is

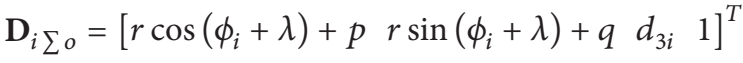

$$
\begin{aligned}
& (i=1,2,3) .
\end{aligned}
$$

As discussed before, the pose of the target plane $\varepsilon$ with respect to the moving plane $\rho$ can be described by the point $t_{2}$ and the normal unit vector $\overrightarrow{\mathbf{t}_{2} \mathbf{w}}$ of the plane $\varepsilon$. Consider

$$
\begin{gathered}
\mathbf{t}_{2 \sum o}=\left[\begin{array}{llll}
0 & 0 & H_{2} & 1
\end{array}\right]^{T}, \\
\overrightarrow{\mathbf{t}}_{\mathbf{2}} \mathbf{w}_{\sum O}=\left[\begin{array}{llll}
\cos \alpha \sin \beta & \sin \alpha \sin \beta & \cos \beta & 0
\end{array}\right]^{T} .
\end{gathered}
$$

Since $\alpha_{1}$ and $\beta_{1}$ can be calculated by (2), the coordinate of vector $\overrightarrow{\mathbf{t}_{2} \mathbf{w}}$ can be transferred from the frame $\Sigma O$ to the frame $\Sigma o$ by Euler's transformation as

$$
\overrightarrow{\mathbf{t}}_{\mathbf{2}} \mathbf{w}_{\sum o}=\left[\begin{array}{cc}
\mathbf{R}^{-1} & \mathbf{0} \\
\mathbf{0} & \mathbf{1}
\end{array}\right] \cdot{\overrightarrow{\mathbf{t}} \mathbf{2} \mathbf{w}_{\sum O}}
$$

$$
\mathbf{R}^{-1}=\left[\begin{array}{lll}
R_{11} & R_{12} & R_{13} \\
R_{21} & R_{22} & R_{23} \\
R_{31} & R_{32} & R_{33}
\end{array}\right]^{-1}=\left[\begin{array}{lll}
r_{11} & r_{12} & r_{13} \\
r_{21} & r_{22} & r_{23} \\
r_{31} & r_{32} & r_{33}
\end{array}\right] \text {, }
$$


TABLE 1: Direct-inverse position analysis of the 3-PSP- $\underline{\mathrm{P}}\left(\mathrm{P}_{\mathrm{H}}\right) \mathrm{HS}-\mathrm{PM}$ measured in frame $\Sigma O\left(R=150, r=55, \varphi_{1}=\psi_{1}=0, \varphi_{2}=\psi_{2}=2 \pi / 3\right.$, $\varphi_{3}=\psi_{3}=10 \pi / 9, p=0, q=0, \lambda=25^{\circ}, \phi_{1}=0^{\circ}, \phi_{2}=90^{\circ}$, and $\left.\phi_{3}=180^{\circ}\right)$.

\begin{tabular}{|c|c|c|c|c|c|c|c|}
\hline Direct & 1 & 2 & 3 & Inverse & 4 & 5 & 6 \\
\hline$d_{11}$ & 2.500000 & 2.200000 & 1.400000 & $\alpha$ & 1.000000 & 3.141593 & 0.500000 \\
\hline$d_{12}$ & 2.000000 & 2.500000 & 2.200000 & $\beta$ & 0.001000 & 0.010000 & 0.020000 \\
\hline$d_{13}$ & 1.500000 & 1.700000 & 2.400000 & $H$ & 2.000000 & 2.500000 & 2.900000 \\
\hline$d_{31}$ & 0.200000 & 0.700000 & 0.400000 & $\alpha_{2}$ & 1.000000 & 0.300000 & 0.010000 \\
\hline$d_{32}$ & 0.500000 & 0.100000 & 0.350000 & $\beta_{2}$ & 0.002000 & 0.005000 & 0.001000 \\
\hline$d_{33}$ & 0.100000 & 0.400000 & 0.500000 & $\mathrm{H}_{2}$ & 0.100000 & 0.800000 & 0.080000 \\
\hline$\alpha$ & -1.735114 & 2.832609 & 0.489347 & $d_{11}$ & 1.546799 & 3.647094 & 0.043040 \\
\hline$\beta$ & 0.007869 & 0.007279 & 0.003988 & $d_{12}$ & 2.076600 & 0.204843 & 2.925715 \\
\hline$H$ & 2.177085 & 2.599314 & 2.369710 & $d_{13}$ & 2.231900 & 0.076335 & 5.936067 \\
\hline$\alpha_{2}$ & 0.933895 & 4.646567 & 0.534729 & $d_{31}$ & 0.170882 & 0.744787 & 0.134401 \\
\hline$\beta_{2}$ & 0.006428 & 0.008624 & 0.002033 & $d_{32}$ & 0.015882 & 1.069403 & 0.071905 \\
\hline $\mathrm{H}_{2}$ & 0.150000 & 0.550000 & 0.450000 & $d_{33}$ & 0.029118 & 0.855213 & 0.025599 \\
\hline$\alpha_{1}$ & -2.669009 & -1.813957 & -0.045381 & $\alpha_{1}$ & 0.000000 & 2.841593 & 0.490000 \\
\hline$\beta_{1}$ & 0.003541 & 0.004172 & 0.003468 & $\beta_{1}$ & 0.002355 & 0.013587 & 0.020979 \\
\hline$H_{1}$ & 2.027085 & 2.049327 & 1.919709 & $H_{1}$ & 1.900000 & 1.699856 & 2.819845 \\
\hline$o_{x}$ & 0.000113 & 0.001052 & -0.000589 & $o_{x}$ & -0.000251 & -0.011449 & 0.004906 \\
\hline$o_{y}$ & 0.000157 & 0.000031 & 0.000040 & $o_{y}$ & 0.000025 & -0.000597 & 0.005566 \\
\hline$o_{z}$ & 2.027085 & 2.049327 & 1.919709 & $o_{z}$ & 1.900000 & 1.699856 & 2.819845 \\
\hline
\end{tabular}

where $r_{11}=\cos \gamma_{1} \cos \beta_{1} \cos \alpha_{1}-\sin \gamma_{1} \sin \alpha_{1} ; r_{12}=$ $\cos \gamma_{1} \cos \beta_{1} \sin \alpha_{1}+\sin \gamma_{1} \cos \alpha_{1} ; r_{13}=-\cos \gamma_{1} \sin \beta_{1}$; $r_{21}=-\cos \gamma_{1} \sin \alpha_{1}-\sin \gamma_{1} \cos \beta_{1} \cos \alpha_{1} ; r_{22}=$ $-\sin \gamma_{1} \cos \beta_{1} \sin \alpha_{1}+\cos \gamma_{1} \cos \alpha_{1} ; r_{23}=\sin \gamma_{1} \sin \beta_{1}$; $r_{31}=\sin \beta_{1} \cos \alpha_{1} ; r_{32}=\sin \beta_{1} \sin \alpha_{1}$; and $r_{33}=\cos \beta_{1}$.

Since the vector $\overrightarrow{\mathbf{t}_{2} \mathbf{D}_{\mathbf{i}}}$ in the plane $\varepsilon$ is orthogonal with respect to the vector $\overrightarrow{\mathbf{t}_{\mathbf{2}} \mathbf{W}}$ in frame $\Sigma o$, the following equation can be obtained:

$$
{\overrightarrow{\mathbf{t}} \mathbf{2} \mathbf{w}_{\sum o}} \cdot{\overrightarrow{\mathbf{t}_{\mathbf{2}} \mathbf{D}_{\mathbf{i} \sum o}}}=0 \quad(i=1,2,3) .
$$

Equation (51) can be rearranged to obtain

$$
\begin{aligned}
d_{3 i}= & H_{2}-\left(p \cdot r_{13}+q \cdot r_{23}\right. \\
& +\left[\left(p \cdot r_{11}+q \cdot r_{12}\right) \cos \alpha\right. \\
& \left.\left.+\left(p \cdot r_{12}+q \cdot r_{22}\right) \sin \alpha\right] \tan \beta\right) \\
& \cdot\left(r_{33}+\left(r_{31} \cos \alpha+r_{32} \sin \alpha\right) \tan \beta\right)^{-1} \\
& -\frac{r \cos \left(\phi_{i}+\lambda\right)\left[r_{13}+\left(r_{11} \cos \alpha+r_{12} \sin \alpha\right) \tan \beta\right]}{r_{33}+\left(r_{31} \cos \alpha+r_{32} \sin \alpha\right) \tan \beta} \\
& +\frac{r \sin \left(\phi_{i}+\lambda\right)\left[r_{23}+\left(r_{21} \cos \alpha+r_{22} \sin \alpha\right) \tan \beta\right]}{r_{33}+\left(r_{31} \cos \alpha+r_{32} \sin \alpha\right) \tan \beta} \quad(i=1,2,3) .
\end{aligned}
$$

The coordinate of the moving platform's central point $o$ in frame $\Sigma O$ can be obtained as shown in Section 4.2. Further, the actuated parameters $d_{1 i}(i=1,2,3)$ of the prismatic joints $\mathrm{P}_{1 i}$ are

$$
\begin{array}{r}
d_{1 i}=o_{z}-\left[\left(R \cos \varphi_{i}-o_{x}\right) \cos \alpha_{1}\right. \\
\left.+\left(R \sin \varphi_{i}-o_{y}\right) \sin \alpha_{1}\right] \tan \beta_{1}, \\
\quad(i=1,2,3) .
\end{array}
$$

With this, the whole actuated parameters of the 3-PSP$\underline{\mathrm{P}}\left(\mathrm{P}_{\mathrm{H}}\right)$ HS-PM in analytical form are obtained.

5.3. Numerical Example. Specify the architectural parameters of the 3-PSP-P $\left(\mathrm{P}_{\mathrm{H}}\right)$ HS-PM according to the definition in Figure 6, as $R=150, r=55, \varphi_{1}=\psi_{1}=0, \varphi_{2}=\psi_{2}=2 \pi / 3$, $\varphi_{3}=\psi_{3}=10 \pi / 9, p=0, q=0, \lambda=25^{\circ}, \phi_{1}=0^{\circ}, \phi_{2}=$ $90^{\circ}$, and $\phi_{3}=180^{\circ}$, which are consistent with the prototype of the IHPRM. Several cases are calculated in both direct and inverse ways of the position analysis, as obtained in Table 1.

The left side of Table 1 shows the position solving in the direct way, which calculate the pose parameters $\alpha, \beta, H, \alpha_{2}$, $\beta_{2}$, and $H_{2}$ with the given actuate parameters $d_{1 i}$ and $d_{3 i}(i=$ $1,2,3)$. The right side of Table 1 shows the position solving in the inverse way, which calculate the actuate parameters $d_{1 i}$ and $d_{3 i}(i=1,2,3)$ with the given pose parameters $\alpha$, $\beta, H, \alpha_{2}, \beta_{2}$, and $H_{2}$. In addition, the moving platform's pose parameters and coordinate of the central point o are also obtained in both sides. The one-to-one correspondent relationships between the actuated parameters and the pose parameters are obvious. 


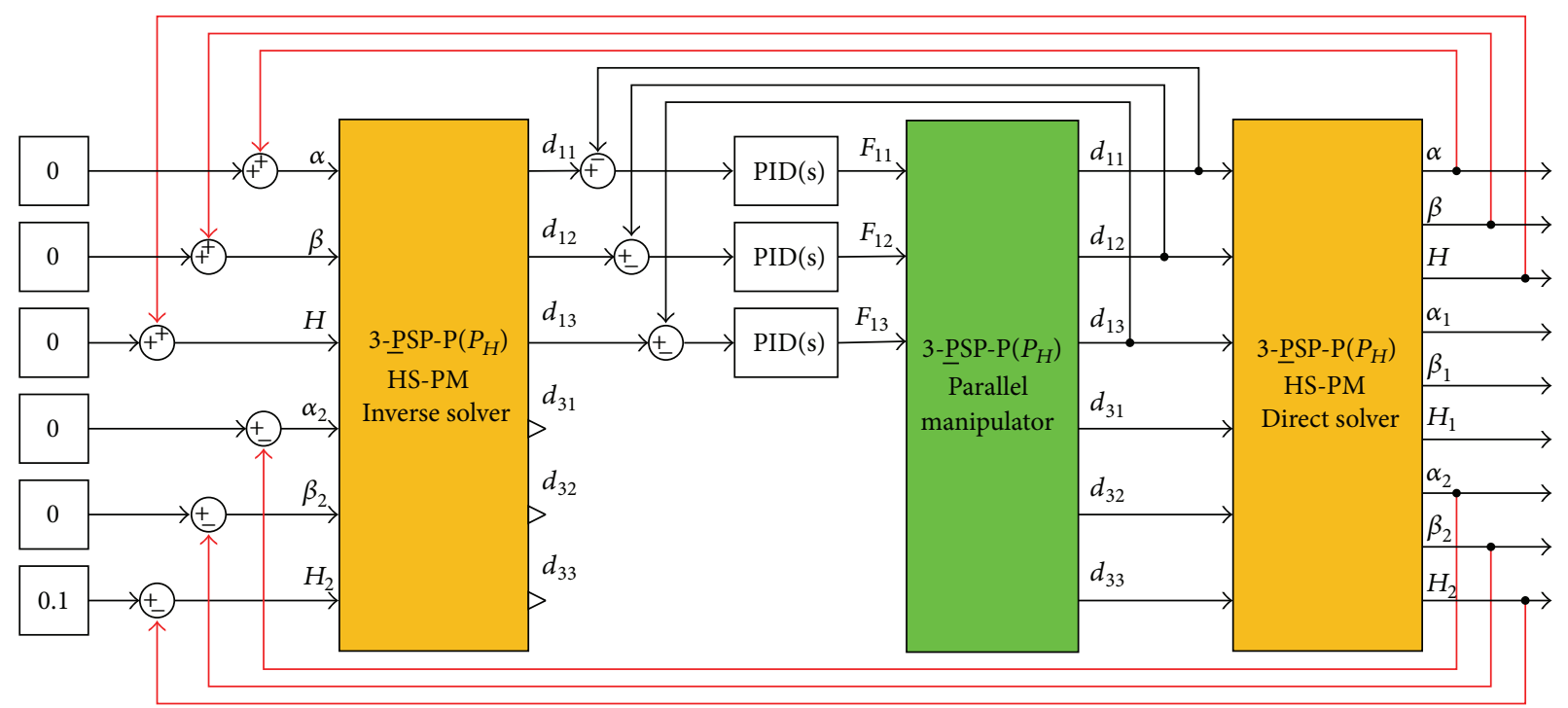

FIGURE 9: The block diagram for the open-loop control method (without the red line) and closed-loop control method (with the red line).

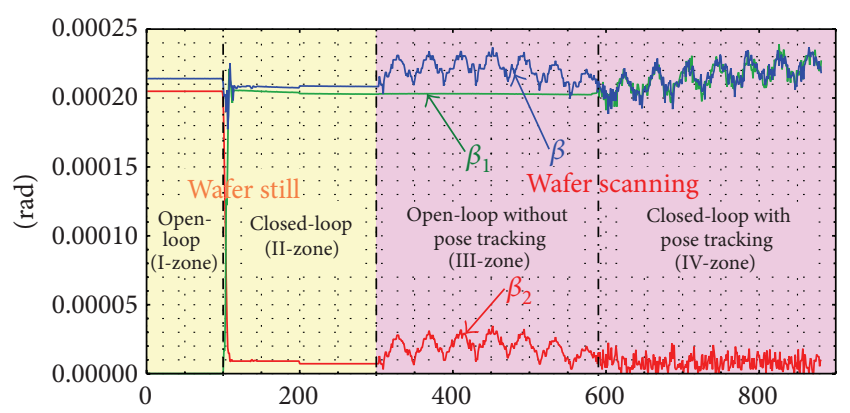

FIGURE 10: The angular parameters during the pose regulating.

\section{Experimental Results and Discussions}

IHPRM regulates the pose of the $\mathrm{IH}$ with $3 \mathrm{DOF}$ and detects the gap status with another 3 DOFs simultaneously. The error caused by the manufacturing and assembly can be reduced efficiently by introducing the closed-loop feedback of the gap status, which can be verified in the prototype.

A series of contrast experiments between the open-loop control method and closed-loop control method are carried out. As shown in Figure 9, the open-loop control method (without the red line) shows the behavior of the single 3PSP PM and assumes that the pose between the wafer surface and the main frame is known and fixed, and the sensors are employed only for the error observation. The closed-loop control method (with the red line) introduces the six pose parameters $\left(\alpha, \beta, H, \alpha_{2}, \beta_{2}\right.$, and $\left.H_{2}\right)$ as the feedback signals.

The experiments are divided into two parts according to the wafer status, as the yellow region and pink region shown in Figures 10 and 11.

The yellow region shows the regulating process of the gap with the wafer still. As shown in the I-Zone (openloop), due to the impact of the deviations generated from the manufacturing and assembly processes, when the IH's lower

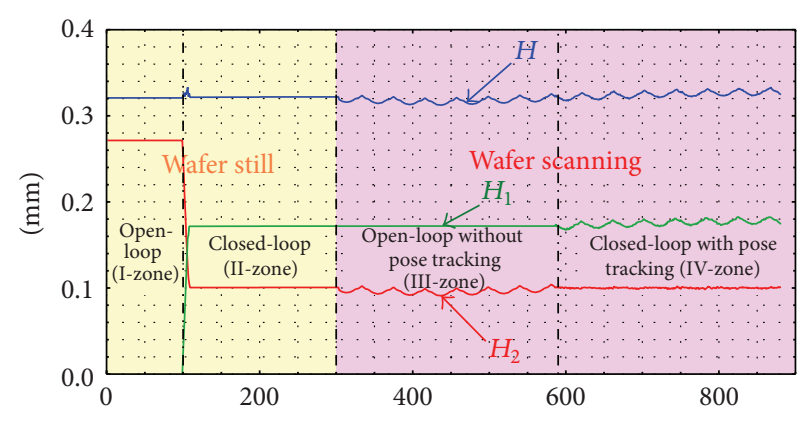

FIGURE 11: The position parameters during the pose regulating.

surface is adjusted to be parallel to the fixed plane, the angle between the IH's lower surface and the wafer surface $\beta_{2}$ is about $0.000205 \mathrm{rad}$ and the central distance $H_{2}$ is about $0.271 \mathrm{~mm}$, which is far beyond the installation requirement of the IH. By introducing the closed-loop feedback of the gap status, the angle $\beta_{2}$ and the central distance $\mathrm{H}_{2}$ are controlled within $0.00001 \mathrm{rad}$ and $0.1 \pm 0.0005 \mathrm{~mm}$, respectively, as shown in the II-Zone (closed-loop). This shows the beneficial effects of the HS-PM in a closed-loop control application.

The pink region shows the variations of the gap with the wafer scanning. The gap status changes violently while the pose of the IH's lower surface remains unchanged during the wafer scanning. As shown in the III-Zone (open-loop control without pose tracking), $\beta_{2}$ varies in the range of $0.000003 \sim$ $0.000035 \mathrm{rad}$ and $H_{2}$ varies in the range of $0.091 \sim 0.105 \mathrm{~mm}$. In order to stabilize the status of the gap, the pose of the IH's lower surface should adjust with respect to the wafer surface in real time, which makes extremely great demands on the computational efficiency of the direct and inverse position analysis. As both the direct and inverse position solutions in analytical form are obtained, the computational efficiency has very big enhancement, making real-time pose 
tracking based on online calculation possible. As shown in the IV-Zone (closed-loop control without pose tracking), by introducing the pose tracking in real time, the angle $\beta_{2}$ and the central distance $\mathrm{H}_{2}$ are controlled within $0.000025 \mathrm{rad}$ and $0.1 \pm 0.002 \mathrm{~mm}$, respectively, during the wafer scanning process, which meets the installation requirements of IH well.

\section{Conclusions}

In this paper, a novel 3- $\underline{\mathrm{PSP}}-\underline{\mathrm{P}}\left(\mathrm{P}_{\mathrm{H}}\right)$ hybrid serial-parallel mechanism is presented to meet the pose regulation requirement of immersion hood in the immersion lithography system. The direct-inverse position analysis as the basic issue of the mechanical study is mainly discussed in this paper. The conclusions are drawn as follows.

(1) By attaching the base platform of the 3- $\underline{P}\left(\mathrm{P}_{\mathrm{H}}\right) \mathrm{PM}$ to the moving platform of the 3-PSP PM, the novel 3-PSP- $\underline{P}\left(\mathrm{P}_{\mathrm{H}}\right)$ HS-PM combines these two parallel modules in serial form and has 6 DOFs.

(2) By choosing the proper pose parameters and constraints, the solutions in analytical form of both 3-PSP PM's direct and inverse position analyses in the couple mixed-type mode are obtained, which improves the analytical analysis of the 3-PSP PM. Based on the position analysis of the 3-PSP PM, both the direct and the inverse position analyses of the 3-PSP-P $\left(\mathrm{P}_{\mathrm{H}}\right)$ HS-PM are discussed, with the solutions presented in analytical form.

(3) Since both the direct and inverse position solutions in analytical form are obtained, the computational efficiency obtains very big enhancement, which makes real-time pose tracking based on online calculation possible. The direct-inverse position model is applied in the real-time control of the IH's lower surface pose tracking with respect to the wafer surface. The stability improvement of the gap is verified by the experiment, which indicates significant effect.

(4) Currently, improving the parallel mechanism's major drawback of the limited workspace while keeping the advantage (precision, rigidity, and load-toweight ratio) is the main focus of many researchers on the hybrid serial-parallel mechanism. The novel 3-PSP- $\underline{P}\left(\mathrm{P}_{\mathrm{H}}\right)$ HS-PM is applied in the immersion hood pose regulation in immersion lithography system. By measuring and regulating the pose of the immersion hood with respect to the wafer surface simultaneously, the immersion hood tracks the pose of the wafer surface in real time, which is another exploration in the applications of the hybrid serialparallel mechanism.

\section{Conflict of Interests}

The authors declare that there is no conflict of interests regarding the publication of this paper.

\section{Acknowledgments}

This work was supported by the Science Fund for Creative Research Groups of National Natural Science Foundation of China (Grant no. 51221004); the National Hi-tech Research and Development Program (863) of China (Grant no. 2012AA040605); and the National Science and Technology Major Projects (Grant no. 2012ZX02701003).

\section{References}

[1] B. J. Lin, "The ending of optical lithography and the prospects of its successors," Microelectronic Engineering, vol. 83, no. 4-9, pp. 604-613, 2006.

[2] J. Mulkens, D. Flagello, B. Streefkerk, and P. Graeupner, "Benefits and limitations of immersion lithography," Journal of Microlithography, Microfabrication and Microsystems, vol. 3, no. 1, pp. 104-114, 2004.

[3] S. Owa and H. Nagasaka, "Immersion lithography: its history, current status, and future prospects," in Lithography Asia 2008, vol. 7140 of Proceedings of SPIE, November 2008.

[4] H. Burnett, T. Shedd, G. Nellis et al., "Control of the receding meniscus in immersion lithography," Journal of Vacuum Science \& Technology B, vol. 23, no. 6, pp. 2611-2616, 2005.

[5] Y. Chen, K.-M. Lee, and X. Fu, "Effect of upper surface characteristics on meniscus stability in immersion flow field," Microelectronic Engineering, vol. 88, no. 8, pp. 1939-1943, 2011.

[6] Y. Chen, K.-M. Lee, C.-Y. Lin, and X. Fu, "Lumped-parameter modeling for meniscus dynamic analysis in immersion flow field," in Proceedings of the 5th Annual Dynamic Systems and Control Conference Joint with the JSME 11th Motion and Vibration Conference (DSCC 2012-MOVIC '12), pp. 535-541, Fort Lauderdale, Fla, USA, October 2012.

[7] Y. Chen, K.-M. Lee, C.-Y. Lin, and X. Fu, "Lumped-parameter modeling of an immersion flow field for analyzing meniscus dynamic behavior," Journal of Dynamic Systems, Measurement and Control, vol. 136, no. 1, Article ID 011001, 2014.

[8] K. J. Waldron, M. Raghavan, and B. Roth, "Kinematics of a hybrid series-parallel manipulation system," Journal of Dynamic Systems, Measurement and Control, Transactions of the ASME, vol. 111, no. 2, pp. 211-221, 1989.

[9] F. Naccarato and P. H. Hughes, "Inverse kinematics of variablegeometry truss manipulators," Journal of Robotic Systems, vol. 8, no. 2, pp. 249-266, 1991.

[10] M. Shahinpoor, "Kinematics of a parallel-serial (hybrid) manipulator," Journal of Robotic Systems, vol. 9, no. 1, pp. 17-36, 1992.

[11] L. Romdhane, "Design and analysis of a hybrid serial-parallel manipulator," Mechanism and Machine Theory, vol. 34, no. 7, pp. 1037-1055, 1999.

[12] T. K. Tanev, "Kinematics of a hybrid (parallel-serial) robot manipulator," Mechanism and Machine Theory, vol. 35, no. 9, pp. 1183-1196, 2000.

[13] D. Kanaan, P. Wenger, and D. Chablat, "Kinematic analysis of a serial-parallel machine tool: the VERNE machine," Mechanism and Machine Theory, vol. 44, no. 2, pp. 487-498, 2009.

[14] O. Ibrahim and W. Khalil, "Inverse and direct dynamic models of hybrid robots," Mechanism and Machine Theory, vol. 45, no. 4, pp. 627-640, 2010.

[15] Q. Zeng and Y.-F. Fang, "Structural synthesis and analysis of serial-parallel hybrid mechanisms with spatial multi-loop 
kinematic chains," Mechanism and Machine Theory, vol. 49, pp. 198-215, 2012.

[16] Q. Zeng and K. F. Ehmann, "Design of parallel hybrid-loop manipulators with kinematotropic property and deployability," Mechanism and Machine Theory, vol. 71, pp. 1-26, 2014.

[17] R.-D. Gregorio and V. Parenti-Castelli, "Position analysis in analytical form of the 3-PSP mechanism," Transactions of the ASME-Journal of Mechanical Design, vol. 123, no. 1, pp. 51-55, 2001.

[18] F. Deng and W.-Z. Li, "Forward solution analysis on spatial positions of 3-PSP parallel mechanism," Journal of Machine Design, no. 1, pp. 41-45, 2009.

[19] Q. Hao, L.-P. Wang, L.-W. Guan, and X.-J. Liu, "Dynamic analysis of a novel 3-PSP 3-DOF parallel manipulator," in Proceedings of the ASME/IFToMM International Conference on Reconfigurable Mechanisms and Robots, pp. 309-314, June 2009.

[20] Z.-L. Zhang and R.-Q. Li, "Analysis on the direct position of 3-PSP parallel mechanism," Machine Design and Research, pp. 225-227, 229, 2013 (Chinese).

[21] A. Rezaei, A. Akbarzadeh, P. M. Nia, and M.-R. AkbarzadehT, "Position, Jacobian and workspace analysis of a 3-PSP spatial parallel manipulator," Robotics and Computer-Integrated Manufacturing, vol. 29, no. 4, pp. 158-173, 2013.

[22] M. Grübler, "Allgemeine Eigenschaften der swangläufigen ebenen kinematische Kette," Civilingenieur, no. 29, pp. 167-200, 1883.

[23] K. Kutzbach, "Einzelfragen aus dem Gebier der Maschinenteile," Zeitschrift der Verein Deutscher Ingenieur, vol. 77, p. 1168, 1993.

[24] Q. Hao, L.-W. Guan, J.-S. Wang, and L.-P. Wang, "Dynamic feedforward control of a novel 3-PSP 3-DOF parallel manipulator," Chinese Journal of Mechanical Engineering, vol. 24, no. 4, pp. 676-684, 2011. 


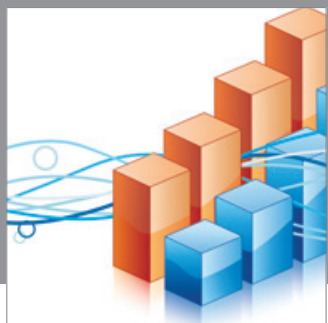

Advances in

Operations Research

mansans

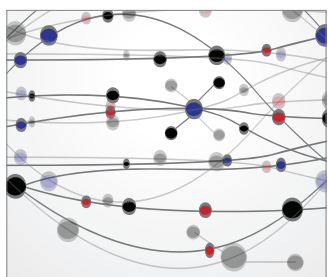

The Scientific World Journal
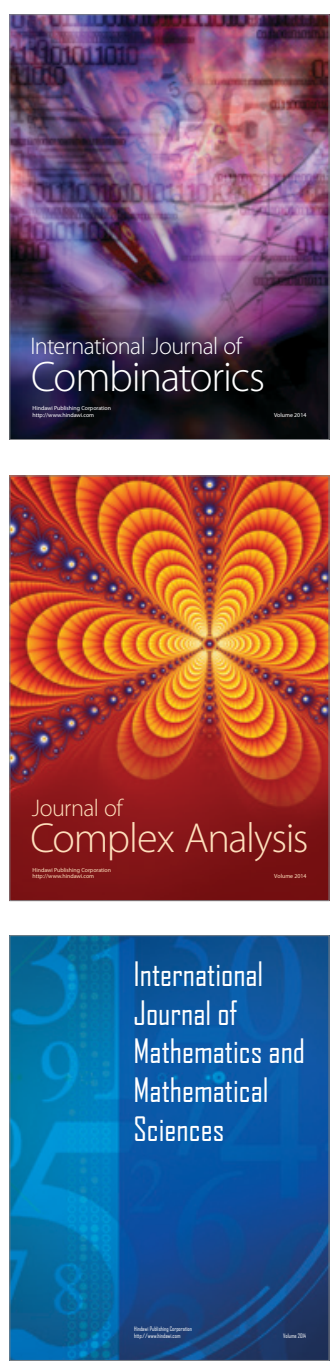
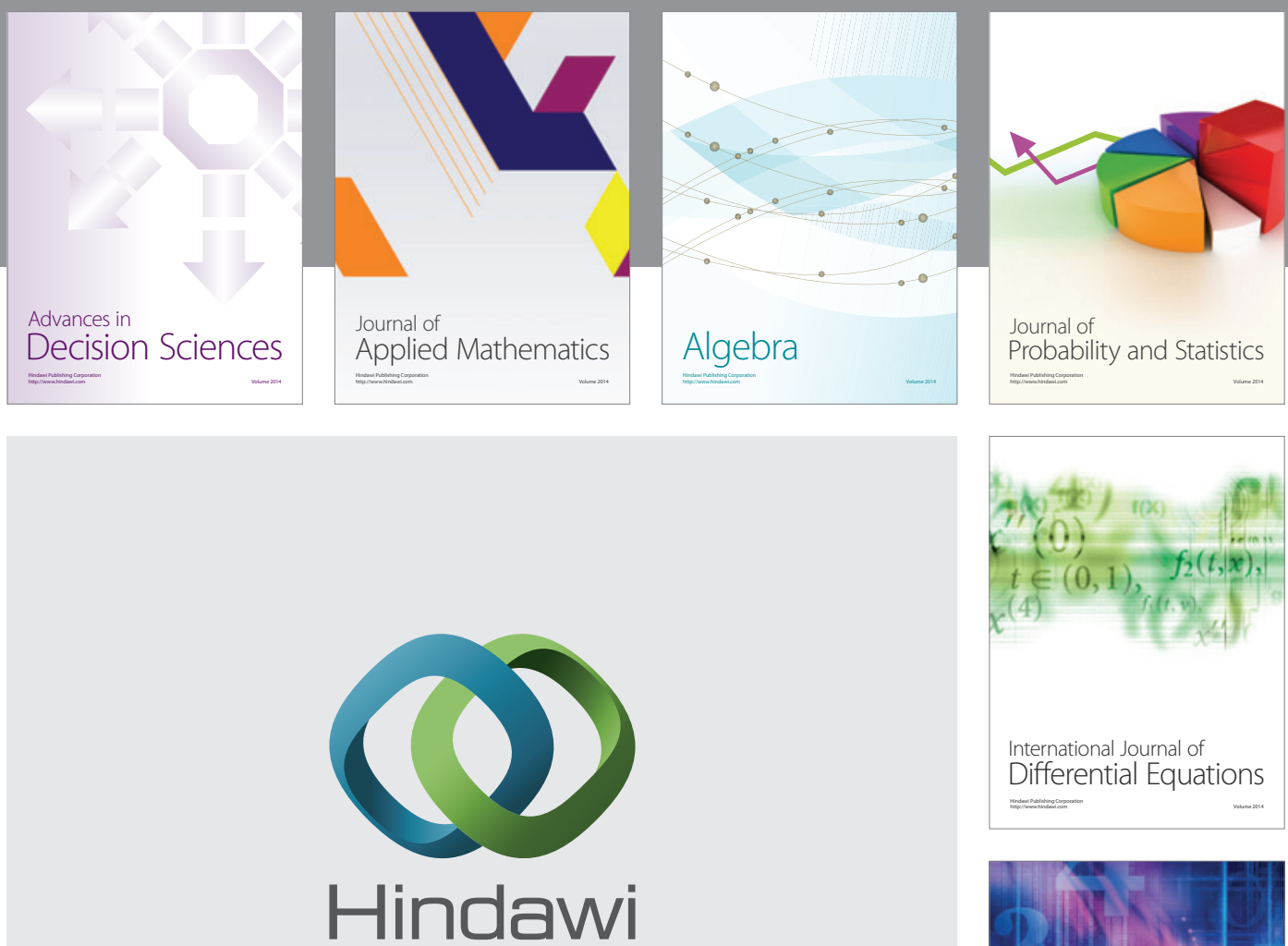

Submit your manuscripts at http://www.hindawi.com
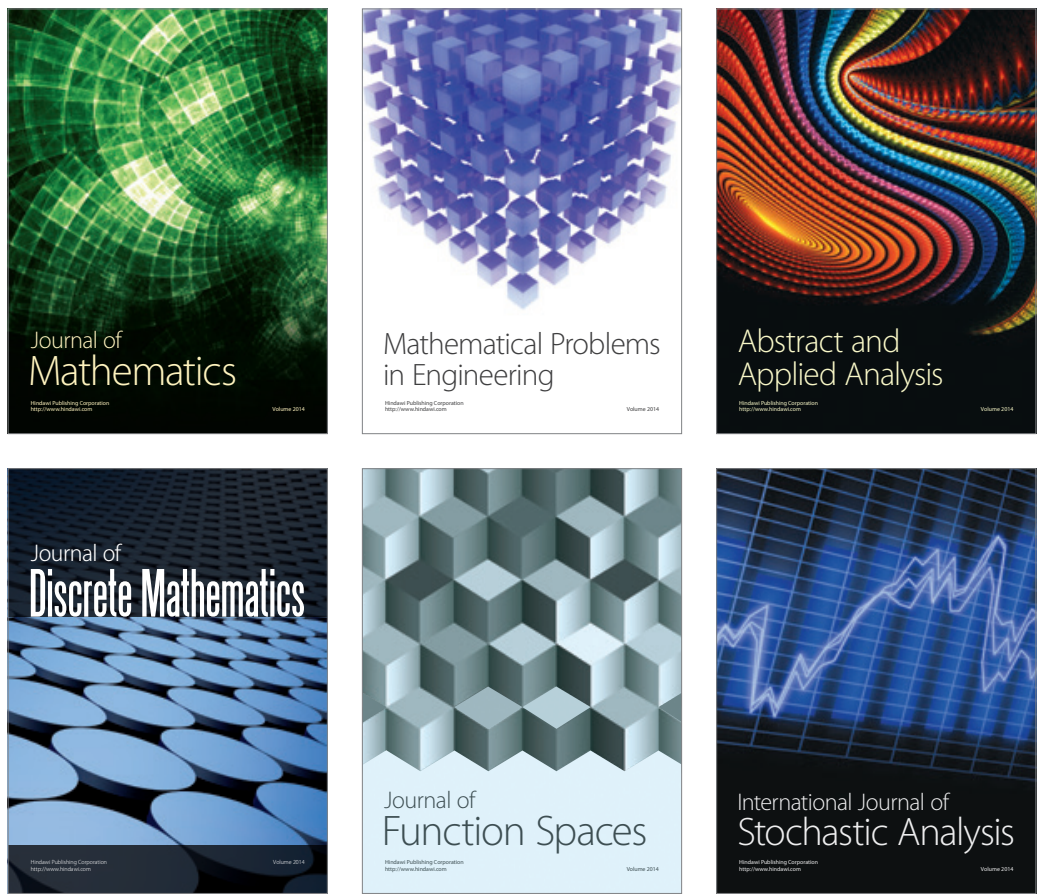

Journal of

Function Spaces

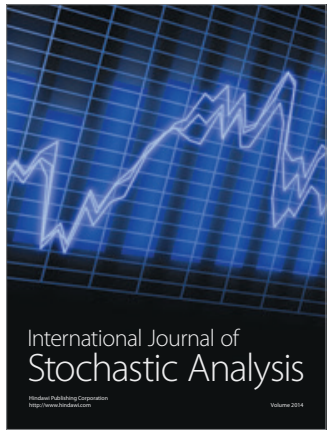

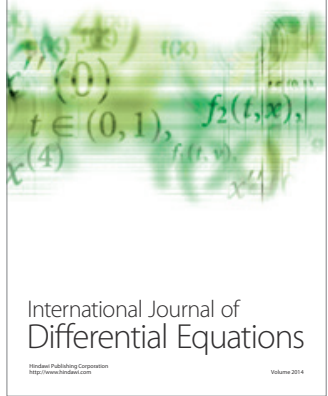
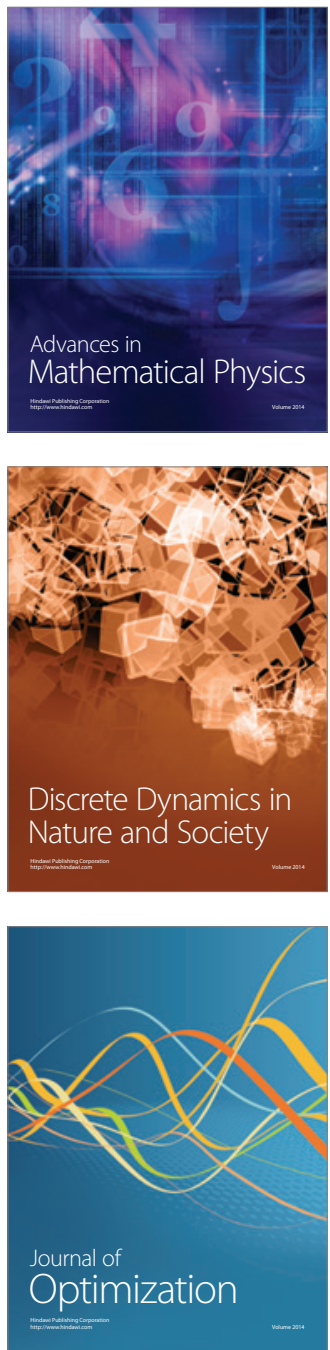